\title{
User-Centric Distributed Spectrum Sharing in Dynamic Network Architectures
}

\author{
Alireza Shams Shafigh ${ }^{\circledR}$, Member, IEEE, Savo Glisic, Senior Member, IEEE, Ekram Hossain ${ }^{\circledR}$, Fellow, IEEE, \\ Beatriz Lorenzo ${ }^{\circledR}$, Member, IEEE, and Luiz A. DaSilva ${ }^{\circledR}$, Fellow, IEEE
}

\begin{abstract}
We develop and analyze a new user-centric networking model for ubiquitous spectrum sharing where every user can share and use the spectrum under uncertainty of their traffic models. In this concept, users when connected to the Internet (wired/wireless) can dynamically serve as access points for other users in their vicinity. For this reason, the concept is referred to as user-centric distributed spectrum sharing. Each user in spectrum sharing mode utilizes a part of its available spectrum for its own traffic and remaining part to share with users in spectrum demanding modes. The model is designed as an operator supervised double-Stackelberg game with network operators, access points, and users as main players. We study network reliability and latency of the system under uncertainty of users' traffic patterns. The numerical results show that the proposed model, depending on different settings, can significantly improve both profit and utility for network operators and users, respectively. Furthermore, network reliability is significantly improved depending on the network parameters for both users and operators.
\end{abstract}

Index Terms-Spectrum sharing, user-centric networking, double Stackelberg game, network reliability.

\section{INTRODUCTION}

$\mathbf{U}$ SER-centric networking models [1]-[3] provide lowcost ubiquitous Internet connectivity without any need for additional fixed infrastructure. Such networking models enable network users to share unused bandwidth with other users [1], [3] especially once users are out of coverage of their operators' networks. A spectrum sharing user (SSU) becomes a resource provider by acting as a dynamic access point and sharing temporally unused resources according to the incentives offered by network operators (NOs) [4], [5]. The concept of dynamic network architecture (DNA) [1] was proposed as an overlay model for pricing and incentives to develop user-centric networks where a user is at the same time

Manuscript received December 20, 2017; revised May 20, 2018 and September 14, 2018; accepted October 17, 2018; approved by IEEE/ACM Transactions on Networking Editor A. Banchs. This work was supported by the WiFiUS Project 243025001/6G-Genesis Flagship of the Academy of Finland. The work of B. Lorenzo was supported by MINECO, Spain, under Grant TEC2016-76465-C2-2-R and Grant EUIN2017-88225. (Corresponding author: Alireza Shams Shafigh.)

A. S. Shafigh and S. Glisic are with the Center for Wireless Communication, Information Technology and Electrical Engineering Faculty, University of Oulu, 90014 Oulu, Finland (e-mail: Alireza.ShamsShafigh@oulu.fi; Savo.Glisic@oulu.fi).

E. Hossain is with the Department of Electrical and Computer Engineering, University of Manitoba, Winnipeg, MB R3T 2N2, Canada (e-mail: Ekram.Hossain@umanitoba.ca).

B. Lorenzo is with the Atlantic Research Center for Telecommunication Technologies, University of Vigo, 36310 Vigo, Spain (e-mail: blorenzo@gti.uvigo.es).

L. A. DaSilva is with Trinity College Dublin, Dublin 2, D02 PN40, Ireland (e-mail: dasilval@tcd.ie).

Digital Object Identifier 10.1109/TNET.2018.2880843 a customer and a provider of network access. The success of DNA relies on active user participation in addition to efficiency of NOs' incentive and pricing mechanisms. DNA as a user-centric networking model enhances network capacity and coverage without extending network fixed infrastructure.

However, previously proposed user-centric networking models [1], [3], [6] rely on deterministic traffic models of users sharing their resources and lack scalability due to the centralized solutions. Unlike these works, this paper introduces a user-centric distributed spectrum sharing (UDSS) model enabling any user throughout the wireless network to either share its resources to assist connections of spectrum demanding users (SDUs) or use the shared resources by spectrum sharing users (SSUs). Such dependable networking paradigm generates a time-varying network topology based on incentive and pricing mechanisms, traffic models and user requirements. Besides, users can reclaim their shared spectrum once they have their own traffic to send that results in interruptions in SDUs' transmissions. These DNA dynamics can be handled by avoiding to choose congested SSUs due to their higher interruption probabilities. SSUs can choose which SDU to interrupt based on its corresponding NO's incentive and pricing mechanisms. In this context, users can operate in three different states of sharing, demanding and idle depending on their traffic requirements and network condition. Every user in SDU mode can ask from both operators and available SSUs for spectrum. Every user in SDU mode can change its state to either SSU to share the unused spectrum with other SDUs or idle not to participate in the sharing process. NOs can control the states of users by developing suitable pricing and incentive mechanisms (PIMs). An idle user (IU) has no interest to participate in the spectrum sharing process since it has either no traffic to send or PIMs do not encourage it to share its resources. We will use the term 'user' to refer to IU, SDU and SSU, otherwise it will be stated explicitly.

A user would choose on its own with whom to share the spectrum and also in which state to operate. For its service, it expects to be reimbursed by the corresponding NOs. By offloading the local traffic from main base stations to local SSUs the overall interference reduces in the network which is in interest of NOs. If the traffic of a SSU increases, it may decide to extend its band and so interrupt a part of transmissions of SDUs already using that band. We define a new metric called SDU transmission interruption probability which indicates the probability that a user in SSU mode reclaims its spectrum shared with SDUs. We elaborate this parameter in order to clarify how efficient is the spectrum sharing process even when users have no prior knowledge about their own traffic. To this end, we include the transmission interruption probability in utility functions and also in pricing and reimbursement mechanisms. 
TABLE I

RELATED WORK

\begin{tabular}{|c|c|c|}
\hline Literature & Contributions & Models/Tools \\
\hline \hline$[9],[10],[11],[12]$ & Commercial user-provided connectivity (UPC) & WiFi or application \\
\hline$[7],[8],[13],[14],[15],[16],[17]$ & Incentive and pricing & Game theory, and optimization \\
{$[18],[19],[20]$} & mechanisms and profit maximization & Survey \\
\hline$[21]$ & Incentive and pricing mechanisms & Game theory,auction, optimization \\
\hline$[13],[14],[22],[23],[24],[25]$ & Crowdsourcing & Game theory and optimization \\
\hline$[3],[26],[27],[28],[29],[30]$ & Topology control and economic models & convex optimization \\
\hline$[31],[32]$ & Opportunistic scheduling & Simulation \\
\hline$[33]$ & Network edge computing \\
\hline$[34]$ & Internet of Things (IoTs) & Game theory and optimization \\
\hline$[6]$ & Spectrum sharing & Optimization and micro economic analysis \\
\hline$[35]$ & Cognitive dynamic network architecture & Software-defined network (SDN) and game theory \\
\hline$[36]$ & Cloud computing & simulation \\
\hline$[37],[38]$ & Security & Mobility prediction and simulation \\
\hline$[39]$ & Mobility Management &
\end{tabular}

The major contributions of this paper can be summarized as follows:

- A new user-centric distributed spectrum sharing model (UDSS) enabled by DNA concept where users act as both the local spectrum consumers and providers.

- A new double Stackelberg game (DSG) is developed to model UDSS in DNA networks.

- An interruption-aware AP selection algorithm for users in the SDU mode to avoid selection of congested APs.

- An incentive/price-based interruption policy (PIP) for each user to force NOs to reduce/increase their prices/ incentives.

- Network reliability and low latency metrics are introduced to show to what extent NOs can rely on DNA to serve their out-of-coverage users.

The rest of the paper is organized as follows: Section II includes related works on user-centric networking models and terminologies of this paper. Section III provides the general system model. Section IV proposes a game theoretic model for UDSS. In Section V, a UDSS model with multiple operators is described in detail. In Section VI, Low Latency and Network Reliability are studied for both single and multi-operator scenarios. Section VII provides numerical results, and finally, Section VIII concludes the paper.

\section{RElated WORK AND TERMinOlOGies}

User-centric networking models [1]-[3], [6] need to be adaptive and distributed in order to cope with network dynamics. The main shortcomings of the previously proposed models are: they lack scalability and do not incorporate any knowledge about traffic model of users. Moreover, these models do not consider dynamics of user-centric spectrum sharing, e.g. interruption probability, access point selection algorithm and spectrum outage probability.

Gao et al. [7] provided a new business model for a singleoperator DNA network. This work is not suitable for DNA networks with multiple operators. In addition, it does not consider spectrum outage, SDU transmission interruption rate and network reliability and latency in the analysis. Interactions between operator and mobile users are formulated by Khalili et al. [8] as a two-stage game. It is shown that the mobile users' capability to act either as regular user or access point can significantly influence the performance of the network. In this work, mobile users' memberships, which depend on traffic patterns, are studied without considering the traffic uncertainty.
Crowdsourcing [13], [14], [22] as an application of DNA in wireless networks enables mobile users to connect with each other and share their Internet connection. In these works, the connectivity is user-initiated and no specific business model is proposed. Afrasiabi and Guerin [15] presented a new insight into the viability of user-provided connectivity services, as well as simple pricing mechanisms to facilitate their successful and effective deployments. Additional works [7]-[39] and their contributions are listed in Table. I. In [9]-[11], hotspots are introduced as movable WiFi-based APs, which users can carry with themselves. NOs motivate users to share these hotspots with other users. The references in Table. I investigate possibilities of applying different techniques, i.e. crowdsourcing, cloud and edge computing, security and mobility management, and cognitive radio networks, to improve DNA performance. With all this in mind, we develop a usercentric distributed spectrum sharing model which is capable to adjust to the traffic dynamics.

Several recent papers propose pricing and incentive mechanisms for DNA [7]-[20]. However, these studies focus on the interaction between host and clients, and none of them considers uncertainty of traffic in their economic model in multiple operator networks. This uncertainty makes the availability of base stations' spectrum unreliable. In this paper, we model this effect through the SDU transmission interruption probability that depends on the uncertainty of the own traffic of SSU users. In addition, the spectrum outage probability and price/incentive-based SDU transmission interruption policy (PIP), introduced in this paper, are the concepts that have not been investigated in the previous works.

\section{A. Terminologies and Definitions}

In this section, we introduce the notations, basic definitions, network architecture, and define explicitly the spectrum sharing mechanism. By exploiting the DNA concept, motivated users might temporary act as user-provided dynamic access points (APs) by sharing their unused spectrum with spectrum demanding users (SDUs) in the network. The spectrum sharing users (SSUs) acting as APs can significantly improve network performance in terms of capacity and coverage. They create a time-varying network topology, where the numbers of spectrum sharing and spectrum demanding users change over time. SSU and SDU are two main operation modes of end-user devices in DNA. In the SSU state, an end-user device can be shared with other end-user devices, which are in the SDU state. A network operator (NO) provides access 
to the Internet by allocating its own Internet connections and can significantly benefit by additionally using user-provided resources. Therefore, strategy of deploying user and operator provided resources by network operators (NOs) results in different network models. First, a NO with fixed network infrastructure (FNI) relies only on pre-established APs (PAPs) to distribute the spectrum between end-user devices without any help from user-provided resources. Second, a NO with fully DNA-based (user-provided) network infrastructure (DNI) deploys only user-provided resources to serve its end-user devices. This type of network relies exclusively on userprovided resources. Third, a NO can simultaneously take advantages of both previous models to create a hybrid network infrastructure (HNI).

In the proposed user-centric distributed spectrum sharing (UDSS) model, interruption probability, sharing and incentive rates are the key parameters in the optimization process. This is because, the sharing process depends on users' traffic and behaviors in addition to NOs' pricing and incentive mechanisms. The NOs and users can enhance their utilities by offering proper incentive $\boldsymbol{\beta}$ and sharing rate $\boldsymbol{\alpha}$.

Definition 1: Interruption probability is the probability that a user in the SSU mode reclaims its shared spectrum from the users in the SDU mode using the spectrum.

Unlike PAPs every user $j$ interrupts SDUs' transmissions with probability $0 \preceq \gamma_{j} \preceq 1$ to transmit their own traffic. This interruption probability depends on the traffic variation of $j$. The interruption probability may significantly degrade the performance of the spectrum sharing mechanism. Thus, it should be considered as a penalty term in the utility function to make SSU users to provide more reliable access for users in the SDU mode. This parameter will be elaborated in more detail later. Every user can make a new contract with NOs to operate in the SSU mode and share its unused bandwidth.

Definition 2: DNA contract for the UDSS is a new type of contract in wireless networks between at least one NO and one network user. The contract includes agreement on how users can use and share the unused bandwidth. It also defines the interruption process, pricing, incentive and penalty mechanisms for the spectrum sharing process.

A DNA contract defines a framework for NOs and users for sharing their spectrum with users in SDU mode. To that end, an acceptable range of the interruption probability for users should be specified in the contracts. The users should be aware that high interruption probability may diminish their benefit. Users might request spectrum from operators through two different DNA contracts: single-operator (SO) and multipleoperator (MO) types [40].

Definition 3: A SO contract defines the required rules for spectrum sharing between users and a single operator. Thus, this contract limits users to share their unused spectrum only with users of a specific NO. On the other hand, an MO contract provides more freedom to users in the spectrum sharing market. A typical user with MO contract can request spectrum from multiple operators and share the unused spectrum with users in the SDU mode belonging to different operators.

In the proposed spectrum sharing model, the spectrum is shared between users through a TDMA scheme. The time for spectrum sharing process is divided into fixed-length timeframes, $t \in\{1,2, \ldots\}$. A time-frame is a set of consecutive time-slots that NO and users can share their spectrum under current network state. The size of a time-frame for userprovided network depends on user-behaviors and their traffic models.

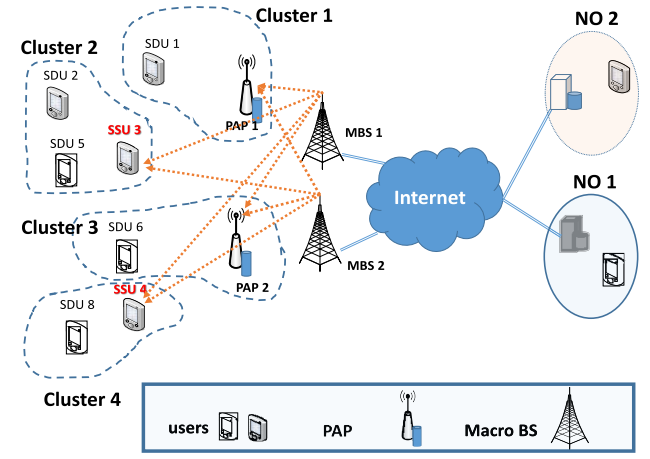

Fig. 1. DNA-enabled wireless network.

Throughout the paper, notations $N$ and $n$ are used to denote the number of operators and index of $n$-th operator, respectively. Furthermore, $\mathcal{N}, \mathcal{J}(t)=\left\{\mathcal{J}_{1}(t), \mathcal{J}_{2}(t), \ldots, \mathcal{J}_{N}(\tau)\right\}$, and $\mathcal{K}(t)=\left\{\mathcal{K}_{1}(t), \mathcal{K}_{2}(t), \ldots, \mathcal{K}_{N}(t)\right\}$ denote the sets of NOs, APs, and SDUs, respectively in time $t$. The $n$-th operator is defined by a tuple $\left(W_{n}, \mathbf{A}_{n}(t), \boldsymbol{\beta}_{n}(t), \mathbf{P}_{n}(t), \mathbf{p}_{n}(t)\right)$, where $\mathbf{A}_{n}(t)=\left\{A_{n, 1}, A_{n, 2}, \ldots, A_{n, J(t)}\right\}, W_{n}=\sum_{j=1}^{J(t)} A_{n, j}$ and $\boldsymbol{\beta}_{n}=\left\{\beta_{n, 1}, \beta_{n, 2}, \ldots, \beta_{n, B_{u}(t)}\right\}$ represent spectrum allocation to APs, total spectrum of NO $n$ and incentive rate for users sharing their unused resources, respectively. Parameters $\mathbf{p}_{n}(t)=\left\{p_{n, 1}, p_{n, 2}, \ldots, p_{n, K(t)}\right\}$ and $\mathbf{P}_{n}(t)=$ $\left\{P_{n, 1}, P_{n, 2}, \ldots, P_{n, B_{u}(t)}\right\}$ represent spectrum prices for users in the SDU and SSU modes, respectively. These prices can significantly change over time according to network state. Throughout the paper, unless stated otherwise explicitly, we use the term AP to refer to both dynamic and preestablished APs. We use PAP and SSU to specifically denote operator-provided AP and user-provided access point, respectively. Therefore, the index $j$ is used for both PAPs and users in SSU modes, or APs in general. To simplify, we use terms SSU and SDU to temporary refer to users in SSU and SDU modes, respectively.

\section{General System Model}

We consider a heterogeneous wireless network as depicted in Fig. 1, where there are $N$ NOs deploying the set $\mathcal{B}^{s}=$ $\left\{\mathcal{B}_{1}^{s}, \mathcal{B}_{2}^{s}, \ldots, \mathcal{B}_{N}^{s}\right\}$ of pre-established (static) access points (PAPs) to serve the set $\mathcal{M}=\left\{\mathcal{M}_{1}, \mathcal{M}_{2}, \ldots, \mathcal{M}_{N}\right\}$ of users, where $\mathcal{B}_{n}^{s}$ and $\mathcal{M}_{n}$ denote the sets of PAPs and users of operator $n$, respectively. In Fig. 1, users are shown in different operation modes. So, there are $\mathcal{J}_{n}(t)=\mathcal{B}_{n}^{s} \cup \mathcal{B}_{n}^{u}(t)$ APs and $\mathcal{K}_{n}(t)=\mathcal{M}-\mathcal{B}_{n}^{u}(t)$ users working in SDU mode in the $n$-th DNA-enabled network in time $t$.

In the proposed user-centric distributed spectrum sharing (UDSS), $A_{n, j}$ is the spectrum shared by NO $n$ with AP $j$. Every PAP $j$ shares all received bandwidth from NOs with SDUs. In DNA network, every SSU $j$ shares its temporary unused spectrum $\sum_{k \in \mathcal{K}(t)} a_{j, k}$ with SDUs, where $a_{j, k}$ is the spectrum shared by SSU $j$ with SDU $k$. The set of SDUs accessing the spectrum of the same SSU $j$ creates cluster $\mathcal{L}_{j}$. We denote the set of SDUs competing with SDU $k$ to access the shared spectrum of $\operatorname{AP} j$ as $\mathcal{L}_{j}(k)$. Every SSU $j$ uses the spectrum $\sum_{n \in \mathcal{N}} A_{n, j}-\sum_{k \in \mathcal{L}_{j}} a_{j, k}$ for its own data, where $\sum_{n \in \mathcal{N}} A_{n, j}$ is the total spectrum acquired from operators and $\sum_{k \in \mathcal{L}_{j}} a_{j, k}$ the total spectrum shared by AP $j$ with SDUs. The problem of authentication of a given SDU when asking access from different operators is similar to the problem of roaming 


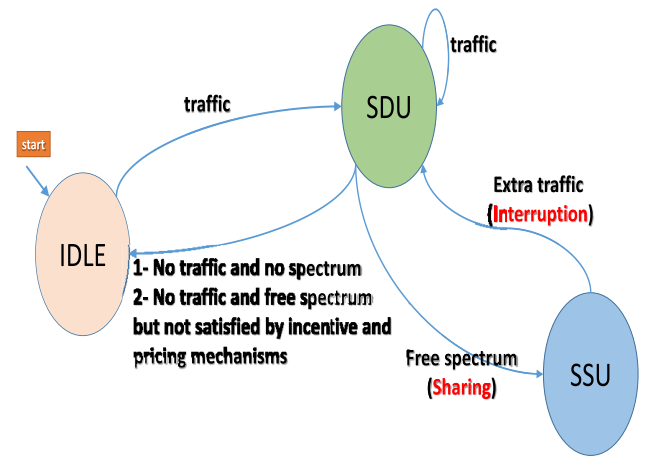

Fig. 2. User states in DNA-enabled wireless networks.

and deserves an additional attention. Due to the limited space it is omitted here.

Utility function of every user $k$ in UDSS can be formulated as

$$
\pi_{k}=\pi_{k}\left[T_{k}^{S S U}\right]+\pi_{k}\left[T_{k}^{S D U}\right]+\pi_{k}\left[T_{k}^{I D L E}\right]
$$

where $T_{k}^{S S U}$ and $T_{k}^{S D U}$ denote the periods of time that user $k$ acts as SSU and SDU, respectively. In (1), $T_{k}^{I D L E}$ represents a period of time that user $k$ does not participate in the spectrum sharing process due to unfavorable pricing and incentive mechanisms. Without loss of generality, we eliminate the third term in (1) and focus on optimizing SSU and SDU modes since users in the idle mode consume power with a constant rate. The life-cycle of user $k$ is represented with $T_{k}$ given as

$$
T_{k}=T_{k}^{S S U}+T_{k}^{S D U}+T_{k}^{I D L E} .
$$

Fig. 2 shows different states of a typical user in a DNAenabled wireless networks, according to incoming traffic, incentive and pricing mechanisms of NOs.

In the sequel, we drop index $t$ from formulations to avoid complexity in notations.

\section{A. User-Centric Distributed Spectrum Sharing (UDSS)}

NOs are the main spectrum suppliers while users also can share their unused spectrum with other users depending on their traffic pattern in addition to pricing and incentive mechanisms. For this reason, there are two different spectrum sharing models in the market between users and APs. The first type is a conventional spectrum sharing model between PAPs and SDUs, and the second one is an opportunistic spectrum sharing model between SSUs and SDUs. The available spectrum in the second one changes with the traffic variations and behavior of user. The NOs motivate SSUs to demand more spectrum than they need for their own traffic by offering them incentives depending on the amount of the spectrum shared by each SSU. The SDUs opportunistically access the shared spectrum by SSUs, which can interrupt the ongoing-transmissions of SDUs. The SSUs have no information in advance about their own traffic variation to explicitly share their bandwidth and avoid interrupting SDU's transmissions. An interruption-aware AP selection (IAS) policy is deployed by SDUs in order to cope with SSUs' traffic uncertainties. The SDUs avoid to choose congested APs since their interruption probability is higher.

We model the interactions among the NOs, APs, and SDUs in both the sharing models with supply-demand concept, where each NO $n$ assigns the band $A_{n, j}$ at the price $P_{n, j}$ to every AP $j$ demanding $D_{j, n}$. Every AP $j$ shares $a_{j, k}$ at price $p_{n, k}$ with SDU $k$ demanding $d_{k, j}$. SSU $j$ shares $a_{j}=\sum_{j^{\prime} \in \mathcal{L}_{j}} a_{j, k}$ out of $\sum_{n \in \mathcal{N}} \min \left(A_{n, j}, D_{j, n}\right)$ with SDUs and uses $\sum_{n \in \mathcal{N}} \min \left(A_{n, j}, D_{j, n}\right)-a_{j}$ for its own data traffic. SDUs request all together $d=\sum_{k \in \mathcal{K}} \sum_{j \in \mathcal{J}} d_{k, j}$ bandwidth from SSUs, where $d_{k, j}$ is the demand of SDU $k$ from SSU $j$. The supplies and demands are equal only in the market equilibrium points, where no player (NO, AP, or SDU) can improve its utility anymore.

The sharing rate of every SSU $j$ in a given time-frame is then calculated by

$$
\alpha_{j}=\frac{\sum_{k \in \mathcal{K}} \min \left(a_{j, k}, d_{k, j}\right)}{\sum_{n \in \mathcal{N}} \min \left(A_{n, j}, D_{j, n}\right)},
$$

where $\min \left(A_{n, j}, D_{j, n}\right)$ and $\min \left(a_{j, k}, d_{k, j}\right)$ ensure that suppliers (NOs and SSUs) cannot share more than their available spectrum and consumers (SSUs and SDUs) do not get more spectrum than their demands. According to (3), every SSU $j$ assigns $\alpha_{j} \sum_{n \in \mathcal{N}} \min \left(A_{n, j}, D_{j, n}\right)$ to SDUs and $\left(1-\alpha_{j}\right) \sum_{n \in \mathcal{N}} \min \left(A_{n, j}, D_{j, n}\right)$ to itself. SSUs regulate the sharing parameters according to the prices and incentive rates. Aforementioned, sharing rate of every PAP $j$ is one since it does not have any traffic of its own to send.

In the next sections, we develop a double Stackelberg game (DSG) with one leader and two group of followers based on standard Stackelberg game model (SGM) [41]-[44] to solve the spectrum sharing problem. In this game, an NO acts as the leader while the APs are the first followers and the SDUs are the second followers.

Note: Notations $\mathbf{X}_{J \times N}$ and $\mathbf{Y}_{J \times K}$ are used throughout the paper to denote the equilibrium points, where $X_{j, n}$ represents the equilibrium point between the demand of SSU $j$ and supply of $n$-th NO, and $Y_{j, k}$ denotes the equilibrium point between the demand of SDU $k$ and supply of SSU $j$.

\section{Double Stackelberg Game (DSG): A Game THEORETIC MODEL}

Here, we consider a scenario with a single NO adjusting prices $\mathbf{P}=\left\{P_{1}, P_{2}, \ldots, P_{J}\right\}$ and $\mathbf{p}=\left\{p_{1}, p_{2}, \ldots, p_{K}\right\}$ for APs and SDUs, respectively, and incentive rate $\boldsymbol{\beta}=$ $\left.\left\{\beta_{1}, \beta_{2}, \ldots, \beta_{J}\right\}\right)$ for SSUs in the network. In sharing mode, every SSU $j$ simultaneously gets the spectrum $\sum_{n \in \mathcal{N}} D_{j, n}$ from NOs and shares $a_{j, k}$ with $k$-th SDU. Furthermore, SDU $k$ gets the spectrum $d_{j, k}$ from AP $j$ according to the price and the SSU's condition (e.g. the offered spectrum, distance, load).

The proposed DSG can be developed in four different steps: 1) NO (as a leader) broadcasts (P, d, $\boldsymbol{\beta})$ to APs. 2) APs, which are the first group of followers, use $(\mathbf{P}, \mathbf{d}, \boldsymbol{\beta})$ to calculate their Nash equilibrium point as the solution of the noncooperative game between APs. They send $(\mathbf{D}, \mathbf{a})$ back to the NO. 3) the NO broadcasts (p,a) to the SDUs. 4) The SDUs as the second followers find their optimum demand $\mathbf{d}$ and send it back to the NO. This procedure continues until all players reach the equilibrium points from which they have no incentive to deviate. Fig. 3-a presents a general framework of a single-operator DNA network with different possible subgames: $\mathrm{NO} \rightarrow \mathrm{APs} \rightarrow$ SDUs.

The backward induction technique (BIT) [45], [46] is used to solve this sequential game. BIT is the process of "looking ahead and working backwards" to solve the subgames based on sequential rationality. BIT in the user-centric spectrum sharing is an iterative process to solve the sub-games sequentially, 


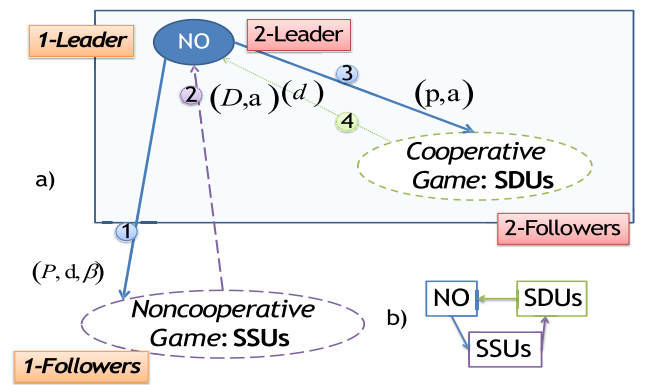

Fig. 3. Double Stackelberg game (DSG) for spectrum sharing in DNA.

where each player (NO or SSU or SDU) optimizes its utility assuming the other players have taken their optimal actions. The process continues in this way backwards in time until all players' actions have been finalized.

\section{A. Single Operator as Leader}

The proposed DSG consists of two separate Stackelberg games with the same leader and different followers. In the first game, the NO is the leader and the APs are the followers. The NO broadcasts $(\mathbf{P}, \mathbf{d}, \boldsymbol{\beta})$, then APs calculate $(\mathbf{D}, \mathbf{a})$. In the second Stackelberg game, the NO is the leader as before and SDUs are followers. Note that the second game takes place just after the first one until all players reach their equilibrium points. Fig. 3-b shows a sequence of actions between different groups of players in DSG.

The profit function of the NO is

$$
\begin{aligned}
\pi(\mathbf{P}, \mathbf{p}, \boldsymbol{\beta})=\sum_{j \in \mathcal{J}} & P_{j}\left(X_{j}-Y_{j}\right) \\
& +\sum_{k \in \mathcal{K}} p_{k} Y_{k}-\sum_{j \in \mathcal{J}} Y_{j} P_{j} \beta_{j}\left(1-\gamma_{j}\right)
\end{aligned}
$$

where $\gamma_{j}$ represents the interruption probability of $\mathrm{AP} j$, $X_{j}=\sum_{n \in \mathcal{N}} X_{j, n}$ and $Y_{j}=\sum_{k \in \mathcal{K}} Y_{j, k}$ denote the demanded and shared spectrum by AP $j$, respectively. Furthermore, $Y_{k}=\sum_{j \in \mathcal{J}} Y_{j, k}$ is the total demanded spectrum by SDU $k$. Every AP $j$ gets $X_{j}$ from the NO and shares $Y_{j}$ with SDUs. According to the definitions, conditions $X_{j} \geq Y_{j}$ and $X_{j}=Y_{j}$ hold for SSU $j$ and PAP $j$, respectively. Inclusion of parameter $\gamma_{j}$ in the utility function forces SSU $j$ to cooperate; otherwise, it diminishes its benefit from the spectrum sharing with SDUs. Note that $\gamma_{j}$ depends on SSU' traffic variation, $\left(\gamma_{j}=0\right.$ for each PAP $j$ ).

For a given incentive rate $\beta$, SSUs obtain a higher effective incentive if they avoid interrupting the ongoing transmission of SDUs, (for each PAP $j, \beta_{j}=0$ ). The interruption probability $\gamma_{j}$ and sharing rate $\alpha_{j}=Y_{j} / X_{j}$ for every AP $j$ are different in (4). According to the definition of PAPs, $\alpha_{j}=1$ for every PAP $j$. We can calculate the interruption probability using the traffic model of every SSU (See Section VI). The NO controls the spectrum sharing mechanism by adjusting $(\mathbf{P}, \mathbf{p}, \boldsymbol{\beta})$.

Under the DSG model, the NO's objective is to maximize its revenue by solving the following optimization problem:

$$
\begin{aligned}
& \text { Problem 1: } \max _{\mathbf{P}, \mathbf{p}, \boldsymbol{\beta}} \pi(\mathbf{P}, \mathbf{p}, \boldsymbol{\beta}) \\
&\text { s.t. } \quad a) \quad \mathbf{P}, \mathbf{p} \succeq 0, \quad b) \quad 0 \preceq \boldsymbol{\beta} \preceq 1,
\end{aligned}
$$

which is a non-convex optimization problem due to the term $\sum_{j \in \mathcal{J}} Y_{j} P_{j} \beta_{j}\left(1-\gamma_{j}\right)$ in the objective function. We can replace the non-convex term $P_{j} \beta_{j}$ with $\frac{1}{4}\left(P_{j}+\beta_{j}\right)^{2}-\frac{1}{4}\left(P_{j}-\right.$ $\left.\beta_{j}\right)^{2}$ to obtain a new objective function of the leader as

$$
\begin{aligned}
\pi(\mathbf{P}, \mathbf{p}, \boldsymbol{\beta})= & \sum_{j \in \mathcal{J}} P_{j}\left(X_{j}-Y_{j}\right) \\
& +\sum_{k \in \mathcal{K}} p_{k} Y_{k}-\frac{1}{4} \sum_{j \in \mathcal{J}} Y_{j}\left(P_{j}+\beta_{j}\right)^{2}\left(1-\gamma_{j}\right) \\
& +\frac{1}{4} \sum_{j \in \mathcal{J}} Y_{j}\left(P_{j}-\beta_{j}\right)^{2}\left(1-\gamma_{j}\right) .
\end{aligned}
$$

The objective function is not in concave form due to the convex term $\left(P_{j}-\beta_{j}\right)^{2}$. We use the first order approximation method to linearize the term $\left(P_{j}-\beta_{j}\right)^{2}$. Note that the higher order terms are not convex and including them in the approximation process increases the complexity. In other words, the higher-order terms are negligible in comparison to the one of the first-order. Besides, the non-convex term is a part of the main objective function. Therefore, the final form of the leader's objective function is given by

$$
\begin{aligned}
\pi(\mathbf{P}, \mathbf{p}, \boldsymbol{\beta})= & \sum_{j \in \mathcal{J}} P_{j}\left(X_{j}-Y_{j}\right) \\
& +\sum_{k \in \mathcal{K}} p_{k} Y_{k}-\frac{1}{4} \sum_{j \in \mathcal{J}} Y_{j}\left(P_{j}+\beta_{j}\right)^{2}\left(1-\gamma_{j}\right) \\
& +\frac{1}{4} \sum_{j \in \mathcal{J}} Y_{j}\left(P_{j}^{(\tau)}-\beta_{j}^{(\tau)}\right)^{2}\left(1-\gamma_{j}\right) \\
& +\frac{1}{2} \sum_{j \in \mathcal{J}} Y_{j}\left\{\left(\left(P_{j}-P_{j}^{(\tau)}\right)-\left(\beta_{j}-\beta_{j}{ }^{(\tau)}\right)\right)\right. \\
& \left.\times\left(P_{j}^{(\tau)}-\beta_{j}^{(\tau)}\right)\right\}\left(1-\gamma_{j}\right),
\end{aligned}
$$

where $\tau$ is the iteration index. Now, the optimization problem is in convex form.

1) APs as the First Followers: At the APs' side, every AP optimizes its own utility function which is given by

$$
\begin{aligned}
\pi_{j}\left(X_{j}, Y_{j}\right)=U_{j}\left(X_{j}, Y_{j}\right) & -P_{j}\left(X_{j}-Y_{j}\right) \\
& +Y_{j} P_{j} \beta_{j}\left(1-\gamma_{j}\right)-E_{j} Y_{j},
\end{aligned}
$$

where the concave function $U_{j}$ defines the SSU $j$ 's benefit from using the spectrum $X_{j}-Y_{j}$ for itself and $E_{j}$ represents the energy consumption function of SSU $j$ due to the spectrum sharing process. The second term $P_{j}\left(X_{j}-Y_{j}\right)$ in (8) is the cost that should be paid by SSU $j$ to the NO. Term $Y_{j} P_{j} \beta_{j}\left(1-\gamma_{j}\right)$ is the reimbursement for SSU $j$ due to the sharing $Y_{j}$ and $Y_{j} P_{j} \gamma_{j}$ is the penalty term due to the unpredicted interruptions caused by SSU $j$ in the spectrum sharing. According to the utility function in (8), every SSU jointly optimizes $X_{j}$ and $Y_{j}$ for the given price and incentive rate. Formally, the optimization problem of every $\operatorname{SSU} j$ can be formulated as

$\begin{aligned} \text { Problem 2: } & \max _{X_{j}, Y_{j}} \pi_{j}\left(X_{j}, Y_{j}\right) \\ & \text { s.t. } \quad \text { a) } \quad \sum_{j \in \mathcal{J}} X_{j} \leq W, \quad \text { b) } \quad Y_{j} \leq X_{j},\end{aligned}$

where constraints (a) and (b) limit the requested spectrum from the NO and the spectrum shared by SSUs. Here, we propose a distributed solution for the SSU optimization problem (9). While in its original form the problem is centrally solved, we can decompose it into subproblems to make it suitable 
for the large scale network. For this reason, we formulate the Lagrangian for the non-cooperative game of SSUs with coupled constraint $\sum_{j \in \mathcal{J}} X_{j} \leq W$ among SSUs as $\ell=\sum_{j \in \mathcal{J}} \pi_{j}-\lambda_{j}\left(X_{j}-W\right)$, where $\lambda_{j}$ is the Lagrange multiplier. The subproblem which should be solved by every $\mathrm{SSU} j$ is given as

$$
\begin{gathered}
\text { Problem 2-1: } G(\boldsymbol{\lambda})=\max _{X_{j}, Y_{j}} \pi_{j}-\lambda_{j}\left(X_{j}-W\right) \\
\text { s.t. } Y_{j} \leq X_{j} .
\end{gathered}
$$

The master problem adjusting $\lambda_{j}$ is defined as

$$
\begin{aligned}
\text { Problem 2-2 : } & \min _{\boldsymbol{\lambda}} \quad G(\boldsymbol{\lambda}) \\
& \text { s.t. } \quad \boldsymbol{\lambda} \succeq 0
\end{aligned}
$$

where $G(\boldsymbol{\lambda})$ is the optimum solutions of optimization problems (10) in terms of $\boldsymbol{\lambda}$. The master problem can be solved iteratively by

$$
\lambda_{j}(\tau+1)=\lambda_{j}(\tau)+\iota \sum_{j \in \mathcal{J}}\left(X_{j}-W\right),
$$

where $\tau$ is the iteration index, $X_{j}$ represents the demand of $\operatorname{SSU} j$ and $\iota$ is the learning rate. For simplicity in notations, we use parameter $\iota$ as learning/step size throughout the paper. The proposed distributed spectrum sharing game for SSUs is developed as Algorithm 1.

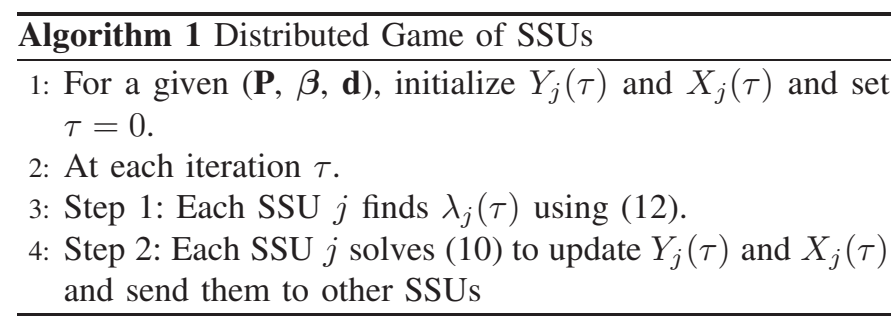

2) Interruption-Aware AP Selection (IAS) Policy by SDUs (Second Followers): Here, we use the basic notations $D_{j}$ and $a_{j}$ for the instant demanded and spectrum shared by SSUs when needed. The SDUs deal with the uncertainty issue by choosing the SSUs with the lowest traffic load in order to have more reliable access. For a given tuple $(\mathbf{a}, \mathbf{p}, \mathbf{X})$, every SDU $k$ maximizes their utility function defined by

$$
\begin{array}{r}
\pi_{k}=\sum_{j \in \mathcal{J}} \ln \left\{q_{j, k}\left(1-\gamma_{j}\right) d_{k, j}\left(1-\left(\theta_{X_{j}}-d_{k, j} / a_{j}\right)\right)\right\} \\
-\sum_{j \in \mathcal{J}} d_{k, j} p_{k} \omega_{j},
\end{array}
$$

where $\omega_{j}$ denotes the cost of renting AP $j$ to use the spectrum $d_{k, j}$, and $q_{j, k}$ is the channel efficiency of SSU $j$ for SDU $k$. If $\mathcal{L}_{j}(k)$ denotes the cluster of SDUs having coupled utility with $\operatorname{SDU} k$, then term $0 \leq \theta_{X_{j}} \leq 1$ is the load index of SSU $j$ and $\sum_{j \in \mathcal{J}} d_{k, j} p_{k}$ is the cost of the requested spectrum $\sum_{j \in \mathcal{J}} d_{k, j}$. The load index $\theta_{X_{j}}$ can be calculated by $\sum_{k^{\prime} \in \mathcal{L} j(k)} d_{k^{\prime}, j} / a_{j}$, where $\sum_{k^{\prime} \in \mathcal{L} j(k)} d_{k^{\prime}, j}$ denotes the aggregated traffic from $\mathcal{L} j(k)$.

In (13), every SSU $j$ is chosen depending on $\sum_{k^{\prime} \in \mathcal{L}_{j}(k)} d_{k^{\prime}, j}$, which is the total demand (load) of SDUs from SSU $j$ in a cluster except SDU $k$. In the above, the term $\sum_{k^{\prime} \in \mathcal{L}_{j}(k)} d_{k^{\prime}, j}$ couples utility functions of SDUs to each other. In order to decompose the coupled term, we assume that every SDU $k$ keeps a local copy of other SDUs' demand as $\mathbf{d}^{(k)}$. Every SDU uses their local copies to locally optimize its demand. To generate the final result, every SDU has to update its local copies throughout the network. We develop the following optimization problem for SDUs:

$$
\begin{aligned}
& \text { Problem 3: } \max _{\mathbf{d}} \sum_{k \in \mathcal{K}} \pi_{k}=-\sum_{k \in \mathcal{K}} \sum_{j \in \mathcal{J}} d_{k, j} p_{k} \\
& \quad+\sum_{k \in \mathcal{K}} \sum_{j \in \mathcal{J}}\left\{\ln \left(q_{j, k}\left(1-\gamma_{j}\right) d_{k, j}\right)\right. \\
& \left.\quad+\ln \left(1-\left(\theta_{X_{j}}-\theta_{X_{k, j}}\right)\right)\right\} \\
& \text { s.t. } a) \sum_{k^{\prime} \in \mathcal{L}_{j}(k)} d_{k^{\prime}, j}^{(k)}+d_{k, j} \leq a_{j}, \forall j \in \mathcal{J}, \\
& \text { b) } d_{k, j}=d_{k, j}^{\left(k^{\prime}\right)}, \forall k, k^{\prime} \in \mathcal{K},
\end{aligned}
$$

where $\theta_{X_{k, j}}=d_{k, j} / a_{j}$ is the sharing index of SSU $j$ to SDU $k$ and the coupled constraint $d_{k, j}=d_{k, j}^{\left(k^{\prime}\right)}$ ensures that all SDUs use the same copies of demands. For this reason, every SDU iteratively sends its demand list around. In the above optimization problem, $d_{k, j}^{\left(k^{\prime}\right)}$ is a local copy of $d_{k, j}$ at SDU $k^{\prime}$. Therefore, the constraint (a) is in term of local variables while the constraint (b) is a coupled constraint. To relax the coupled constraint in (14) we apply a dual decomposition [47], [48] approach by forming Lagrangian as:

$$
\ell=\sum_{k \in \mathcal{K}} \pi_{k}-\sum_{k \in \mathcal{K}} \sum_{j \in \mathcal{J}} \zeta_{k, j} \sum_{k^{\prime} \in \mathcal{L}_{j}(k)}\left(d_{k, j}-d_{k, j}^{\left(k^{\prime}\right)}\right),
$$

where $\zeta_{k, j}$ is a dual variable. The Lagrangian is separated into $K$ sub-problems such that the local version of the optimization problem that should be run by every $\operatorname{SDU} k$ is given as

$$
\begin{array}{r}
\text { Problem 3-1 : } \max _{\mathbf{d}} \pi_{k}-\sum_{j \in \mathcal{J}} \zeta_{k, j} \sum_{k^{\prime} \in \mathcal{L}_{j}(k)}\left(d_{k, j}-d_{k, j}^{\left(k^{\prime}\right)}\right) \\
\text { s.t. } \sum_{k^{\prime} \in \mathcal{L}_{j}(k)} d_{k^{\prime}, j}^{(k)}+d_{k, j} \leq a_{j}, \forall j \in \mathcal{J} .
\end{array}
$$

The master problem can be solved iteratively by subgradient method at each SDU $k$ by

$$
\zeta_{k, j}(\tau+1)=\zeta_{k, j}(\tau)-\iota \sum_{k^{\prime} \in \mathcal{L}_{j}(k)}\left(d_{k, j}-d_{k, j}^{\left(k^{\prime}\right)}\right) .
$$

Thus, every SDU $k$ locally solves (16) and (17) to update its demand $\sum_{j \in \mathcal{L}_{j}(k)} d_{k, j}$. The optimization problem in (14) can be solved by Algorithm 2.

\begin{tabular}{l}
\hline Algorithm 2 Interruption-Aware AP Selection Policy (IAS) \\
\hline 1: At each iteration $\tau$. \\
2: Step 1: each SDU $k$ updates $\zeta$ by (17) \\
3: Step 2: each SDU $k$ locally solves the problem in (16). \\
4: Step 3: each SDU $k$ broadcasts its demand. \\
\hline
\end{tabular}

3) Single Operator Subgame Perfect Equilibrium (SOSPE) of DSG: Problems (5), (9) and (14) create two embedded Stackelberg games where the objective of these games is to find the subgame perfect equilibrium (SPE) points.

Proposition 1: The equilibrium point for the first Stackelberg game is the Nash equilibrium of the game between NO and SSUs while in the second Stackelberg game the Nash equilibrium (NE) is between NO and SDUs. 
Proof: The proof is straightforward.

Proposition 2: A strategy profile is a subgame perfect equilibrium (SPE) of DSG if it is a Nash equilibrium of every subgame of DSG.

Proof: The proof is straightforward. The proposed DSG includes different subgames among NO, SDUs and SSUs. Therefore, the SPE of DSG is the equilibrium point where players including NO, SDUs, and SSUs have no incentives to leave this point; otherwise, at least one of the players is not in its equilibrium point.

Theorem 1: Let $\boldsymbol{P}, \boldsymbol{p}$, and $\boldsymbol{\beta}$ be a solution of (5), $D_{j}$ and $a_{j}$ be a solution of (9) for every SSU $j$ and $\boldsymbol{d}$ be a solution of (14). Then the point $\left(\boldsymbol{P}^{*}, \boldsymbol{p}^{*}, \boldsymbol{\beta}^{*}, \boldsymbol{D}^{*}, \boldsymbol{a}^{*}, \boldsymbol{d}^{*}\right)$ is a SPE point for DSG if for any possible solution $(\boldsymbol{P}, \boldsymbol{p}, \boldsymbol{\beta}, \boldsymbol{D}, \boldsymbol{a}, \boldsymbol{d})$, the following conditions hold:

$$
\begin{array}{ll}
\text { a) } & \pi\left(\boldsymbol{P}^{*}, \boldsymbol{p}^{*}, \boldsymbol{\beta}^{*}, \boldsymbol{D}^{*}, \boldsymbol{a}^{*}, \boldsymbol{d}^{*}\right) \geq \pi\left(\boldsymbol{P}, \boldsymbol{p}, \boldsymbol{\beta}, \boldsymbol{D}^{*}, \boldsymbol{a}^{*}, \boldsymbol{d}^{*}\right) \\
\text { b) } \pi_{j}\left(\boldsymbol{P}^{*}, \boldsymbol{\beta}^{*}, \boldsymbol{D}_{j}^{*}, \boldsymbol{a}_{j}^{*}, \boldsymbol{D}_{-j}^{*}, \boldsymbol{a}_{-j}^{*}, \boldsymbol{d}^{*}\right) \geq \\
& \pi_{j}\left(\boldsymbol{P}^{*}, \boldsymbol{\beta}^{*}, \boldsymbol{D}_{j}, \boldsymbol{a}_{j}, \boldsymbol{D}_{-j}^{*}, \boldsymbol{a}_{-j}^{*}, \boldsymbol{d}^{*}\right), \forall j \in \mathcal{J} \\
\text { c) } & \sum_{k \in \mathcal{K}} \pi_{k}\left(\boldsymbol{p}^{*}, \boldsymbol{a}^{*}, \boldsymbol{d}^{*}\right) \geq \sum_{k \in \mathcal{K}} \pi_{k}\left(\boldsymbol{p}^{*}, \boldsymbol{a}^{*}, \boldsymbol{d}\right) .
\end{array}
$$

Proof: Inequality $(a),(b)$, and (c) imply that the leader (NO) and the followers (SDUs or SSUs) choose their optimum strategy to obtain their market equilibrium points. The constraint $(a)$ emphasizes that the NO runs its SPE strategy and it does not deviate from this state because it is in the optimum point. Likewise, the constraint $(b)$ and $(c)$ define the equilibrium point of SSUs and SDUs, respectively. Note that, in the equilibrium points, the supplies and demands are equal and players will not leave these points.

In (SOSPE), $\mathbf{D}_{-j}^{*}$ and $\mathbf{a}_{-j}^{*}$ define the optimum strategies of all SSUs except $j$ in the demanding and sharing phases, respectively.

Theorem 2: For the proposed DSG, there is a unique SPE.

Proof: We use three convex optimization problems to maximize utilities of NO, SDUs, and SSUs. One can see that the optimization problem is in convex form. Thus, there is optimum solution for each one. According to (SOSPE), every player finds its optimum solution. Therefore, the game has a unique SPE.

In the proposed DSG, the SPE point can be achieved by iteratively solving problems 1,2, and 3. The proposed DSG is developed as Algorithm 3.

Algorithm 3 Double Stackelberg game (DSG) for Distributed Spectrum Sharing

1: Initialize $\mathbf{a}(\tau), \mathbf{D}(\tau)$ and $\mathbf{d}(\tau)$ and set $\tau=0$.

2: At each iteration $\tau$.

3: Step 1) Leader1 (NO) solves (5) to update $(\mathbf{P}(\tau), \mathbf{p}(\tau)$, $\boldsymbol{\beta}(\tau))$ and broadcast $(\mathbf{P}(\tau), \mathbf{p}(\tau), \boldsymbol{\beta}(\tau), \mathbf{a}(\tau), \mathbf{D}(\tau))$.

4: Step 2) Every SSU $j$ (the first followers) solves (10) to update $(\mathbf{a}(\tau), \mathbf{D}(\tau))$ and send it back to the Leader 1 .

5: Step 3) Leader2 (NO) broadcasts $(\mathbf{p}(\tau), \mathbf{a}(\tau))$ to SDUs.

6: Step 4) The second followers (SDUs) solve (9) to update $\mathbf{d}(\tau)$ and return it back to the Leader2.

In the proposed game, the NO is the leader in both Stackelberg games.

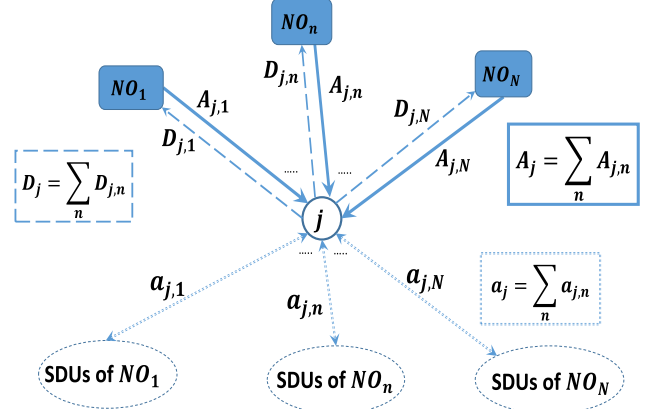

Fig. 4. SSU $j$ with MO contract in the spectrum sharing model.

\section{DYNAMIC NETWORK ARCHITECTURE With MUltiple OPERATORS}

\section{A. Spectrum Sharing in a Multiple Operator Scenario}

In this section, we consider a multiple-operator DNA network [3], where $N$ operators deploy the DNA spectrum sharing model to serve their users. Fig. 4 shows a typical user $j$ with $\mathrm{MO}$ contract interacting with SDUs and NOs. We use the scheme shown in Fig. 4 to convert a threelevel (NOs $\rightarrow$ SSUs $\rightarrow$ SDUs) to a two-level spectrum sharing model (NOs $\rightarrow$ SSUs). This conversion requires some changes in the notations used so far. We assume that the SDUs accept the whole spectrum shared by SSUs in order to focus on interactions between the SSUs and NOs. Therefore, the indices of NOs can be used to denote their user sets. The SSUs get spectrum from different NOs and share it with the SDUs belonging to different operators. The NOs adjust the price and incentive rate based on the amount of demand and spectrum shared by every SSU. According to Fig. 4, we define matrix $y$ in order to explicitly show the structure of the spectrum shared by users. We assume that every entry $y_{j, n}$ of $y_{J \times N}$ denotes the spectrum shared by SSU $j$ with the set of all SDUs belonging to NO $n$.

Every NO $n$ shares its spectrum $\mathbf{A}_{n}$ directly with APs and indirectly with SDUs. The NOs obtain revenue from serving users but they have to give reimbursements to motivate the SSUs inversely proportional to their interruption probabilities. We formulate the interactions between the NOs and SSUs as a Stackelberg game with multiple leaders and multiple followers. This Stackelberg game consists of two subgames. The first game is a cooperative game among the NOs optimizing sum of their payoffs for a given demand from users. The second game is a non-cooperative game among the SSUs maximizing individually their own utility for the given prices and incentive rates.

\section{B. NOs as Leaders}

Let us define the profit function for the main spectrum suppliers (NOs) as (18), as shown at the top of the this page, where $P_{n, n^{\prime}}$ represents the prices set by NO $n$ for SSUs and SDUs of NO $n^{\prime}$ in a multiple-operator DNA network. In (18), the term $\sum_{j \in \mathcal{J}_{n}} \sum_{n^{\prime} \in \mathcal{N}} P_{n, n^{\prime}} y_{j, n^{\prime}}$ is the revenue of NO $n$ from SDUs using the spectrum shared by its APs in $\mathcal{J}_{n}$. The second term $\sum_{n^{\prime} \in \mathcal{N}} \sum_{j \in \mathcal{J}_{n^{\prime}}} P_{n, n^{\prime}} X_{j, n}$ represents the revenue of $n$ th NO from spectrum sharing with APs belonging to different operators. The third term $\sum_{n^{\prime} \in \mathcal{N} \backslash n} \sum_{j \in \mathcal{J}_{n^{\prime}}} P_{n^{\prime}, n} y_{j, n}$ is paid by the $n$-th NO to APs in $\mathcal{J}$ for sharing spectrum with its SDUs. The amount of reimbursement by NO $n$ for SSU $j \in \mathcal{J}_{n^{\prime}}, \forall n^{\prime} \in \mathcal{N}$ is denoted by $P_{n, n^{\prime}} \beta_{n, j}\left(1-\gamma_{j, n}^{\prime}\right) y_{j, n}$, where 


$$
\Pi_{n}\left(\mathbf{P}_{n}, \boldsymbol{\beta}_{n}\right)=\sum_{j \in \mathcal{J}_{n}} \sum_{n^{\prime} \in \mathcal{N}} P_{n, n^{\prime}} y_{j, n^{\prime}}+\sum_{n^{\prime} \in \mathcal{N}} \sum_{j \in \mathcal{J}_{n^{\prime}}} P_{n, n^{\prime}} X_{j, n}-\sum_{n^{\prime} \in \mathcal{N} \backslash n} \sum_{j \in \mathcal{J}_{n^{\prime}}} P_{n^{\prime}, n} y_{j, n}-\sum_{n^{\prime} \in \mathcal{N}} \sum_{j \in \mathcal{J}_{n^{\prime}}} P_{n, n^{\prime}} \beta_{n, j}\left(1-\gamma_{j, n}^{\prime}\right) y_{j, n}
$$

$\gamma_{j, n}^{\prime}$ is the interruption probability of NO $n$ 's users by SSU $j$. Every SSU interrupts SDUs' transmission with different probability depending on the price and incentive rate of their corresponding NOs. This interruption probability in a multioperator scenario enables SSUs to motivate the NOs to reduce their prices or increase their incentive rates. Note that, for serving out-of-coverage SDUs, the NOs rely on SSUs. For this reason, $\gamma_{j, n}^{\prime}$ is an important parameter in a multiple-operator scenario controlled by the offered price and incentive rate of the NOs.

Proposition 3: Every $S S U j \in \mathcal{J}_{n^{\prime}}$ of $N O n^{\prime}$ deploys a price and incentive-aware interruption policy $(P I P)$ to reclaim the allocated spectrum to SDUs of NO $n$ with probability $\gamma_{j, n}^{\prime}$ defined as

$$
\gamma_{j, n}^{\prime}=\gamma_{j} \frac{\frac{P_{n, n^{\prime}}}{\beta_{n, j}}}{\sum_{n^{\prime \prime} \in \mathcal{N}} \frac{P_{n^{\prime \prime}, n^{\prime}}}{\beta_{n^{\prime \prime}, j}}},
$$

where $\gamma_{j}$ is the average packet arrival probability of SSU $j$. Using PIP, the SSUs would interrupt the SDUs belonging to high-price and low-incentive operators with higher probability. For the simplicity in notation we assume that the SSUs only consider price in their interruption policy. Therefore, the last term of (18) is reformulated as

$$
\sum_{n^{\prime} \in \mathcal{N}} \sum_{j \in \mathcal{J}_{n^{\prime}}} P_{n, n^{\prime}} \beta_{n, j}\left(1-\gamma_{j} \frac{P_{n, n^{\prime}}}{\sum_{n^{\prime \prime} \in \mathcal{N}} P_{n^{\prime \prime}, n^{\prime}}}\right) y_{j, n}
$$

With all this in mind, the first game can be formulated as

$$
\begin{aligned}
\text { Problem } 4: & \max _{\mathbf{P}, \boldsymbol{\beta}} \Pi_{n}\left(\mathbf{P}_{n}, \boldsymbol{\beta}_{n}\right) \\
& \text { s.t. } a) \mathbf{P}_{n} \succeq 0, \quad \text { b) } 0 \preceq \boldsymbol{\beta}_{n} \preceq 1,
\end{aligned}
$$

where $\mathbf{P}_{N \times N}$ and $\boldsymbol{\beta}_{N \times J}$ are the price and incentive vectors, respectively. The problem should be centrally solved in its original form due to the coupled pricing term in the objective (20). Every NO requires to know about other NOs' pricing to be able to optimize its revenue. We use dual decomposition approach [47]-[49] to develop a distributed pricing game among NOs. In this approach, the NOs constantly exchange their prices in order to optimize their profit. We reformulate the objective function in (18) as

$$
\begin{aligned}
\hat{\Pi}_{n}\left(\mathbf{P}_{n}, \boldsymbol{\beta}_{n}\right)= & \sum_{j \in \mathcal{J}_{n}} \sum_{n^{\prime} \in \mathcal{N}} P_{n, n^{\prime}} y_{j, n^{\prime}}+\sum_{n^{\prime} \in \mathcal{N}} \sum_{j \in \mathcal{J}_{n^{\prime}}} P_{n, n^{\prime}} X_{j, n} \\
& -\sum_{n^{\prime} \in \mathcal{N}, n^{\prime} \neq n} \sum_{j \in \mathcal{J}_{n^{\prime}}} P_{n^{\prime}, n}^{(n)} y_{j, n} \\
& -\sum_{n^{\prime} \in \mathcal{N}} \sum_{j \in \mathcal{J}_{n^{\prime}}} P_{n, n^{\prime}} \beta_{n, j} y_{j, n} \\
& +\sum_{n^{\prime} \in \mathcal{N}} \sum_{j \in \mathcal{J}_{n^{\prime}}} \frac{P_{n, n^{\prime}}^{2} \beta_{n, j} \gamma_{j}}{\sum_{n^{\prime \prime} \in \mathcal{N}, n^{\prime \prime} \neq n} P_{n^{\prime \prime}, n^{\prime}}^{(n)}+P_{n, n^{\prime}}} y_{j, n},
\end{aligned}
$$

and the optimization problem (20) as

$$
\begin{aligned}
\text { Problem 4-1 : } & \max _{\mathbf{P}, \boldsymbol{\beta}} \\
\text { s. t. } & \hat{\Pi}_{n}\left(\mathbf{P}_{n}, \boldsymbol{\beta}_{n}\right) \\
& \text { a) } \mathbf{P} \succeq 0, \\
& \text { c) } 0 P_{n, n^{\prime}}=P_{n, n^{\prime}}^{\left(n^{\prime}\right)}, \forall n^{\prime} \in \mathcal{N},
\end{aligned}
$$

where $P_{n, n^{\prime}}^{\left(n^{\prime}\right)}$ represents a local copy of price $P_{n, n^{\prime}}$ seen by NO $n^{\prime}$. In the proposed distributed method, every NO constantly keeps a local copy of other NOs' prices and sends its price to other NOs. For consistency, the local copies of prices need to be equal to the real ones $\left(P_{n, n^{\prime}}=P_{n, n^{\prime}}^{\left(n^{\prime}\right)}\right.$ ). The objective function in (22) is in terms of local variables except the coupled constraint (c). To solve (22), we again use the dual decomposition approach. In this process we also use inequality $e^{f_{n, n^{\prime}, j}} \leq P_{n, n^{\prime}} \beta_{n, j}$ and

$$
e^{g_{n, n^{\prime}, j}} \leq \frac{P_{n, n^{\prime}}^{2} \beta_{n, j}}{\sum_{n^{\prime \prime} \in \mathcal{N}, n^{\prime \prime} \neq n} P_{n^{\prime \prime}, n^{\prime}}^{(n)}+P_{n, n^{\prime}}}
$$

to approximate the non-convex terms in objective function. We can reformulate (22) for each NO $n$ as

$$
\text { Problem 4-2: } \begin{aligned}
\max _{\mathbf{P}_{n}, \boldsymbol{\beta}_{n}, \mathbf{f}_{n}, \mathbf{g}_{n}} & \sum_{j \in \mathcal{J}_{n}} \sum_{n^{\prime} \in \mathcal{N}} P_{n, n^{\prime}} y_{j, n^{\prime}} \\
& +\sum_{n^{\prime} \in \mathcal{N}} \sum_{j \in \mathcal{J}_{n^{\prime}}} P_{n, n^{\prime}} X_{j, n} \\
& -\sum_{n^{\prime} \in \mathcal{N}, n^{\prime} \neq n} \sum_{j \in \mathcal{J}_{n^{\prime}}} P_{n^{\prime}, n}^{(n)} y_{j, n} \\
& -\sum_{n^{\prime} \in \mathcal{N}} \sum_{j \in \mathcal{J}_{n^{\prime}}} e^{f_{n, n^{\prime}, j}} y_{j, n} \\
& +\sum_{n^{\prime} \in \mathcal{N}} \sum_{j \in \mathcal{J}_{n^{\prime}}} e^{g_{n, n^{\prime}, j} \gamma_{j} y_{j, n}} \\
& -\sum_{n^{\prime} \in \mathcal{N} \backslash n} \chi_{n, n^{\prime}}\left(P_{n, n^{\prime}}-P_{n, n^{\prime}}^{\left(n^{\prime}\right)}\right) \\
\text { s.t. a) } & \mathbf{P}_{n} \succeq 0, \\
\text { b) } & 0 \preceq \boldsymbol{\beta}_{n} \preceq 1, \\
c) & f_{n, n^{\prime}, j} \leq \ln P_{n, n^{\prime}}+\ln \beta_{n, j}, \\
\text { d) } & g_{n, n^{\prime}, j} \leq 2 \ln P_{n, n^{\prime}}+\ln \beta_{n, j} \\
& -\ln \sum_{n^{\prime} \in \mathcal{N} \backslash n} P_{n, n^{\prime}}^{(n)} \\
& -\ln \left(\sum_{n^{\prime \prime} \in \mathcal{N}, n^{\prime \prime} \neq n} P_{n^{\prime \prime}, n^{\prime}}^{(n)}+P_{n, n^{\prime}}\right),
\end{aligned}
$$

where $\chi_{n, n^{\prime}}$ is Lagrange multiplier for inconsistency of prices and can be updated by

$$
\chi_{n, n^{\prime}}(\tau+1)=\left[\chi_{n, n^{\prime}}(\tau)-\iota\left(\sum_{n^{\prime} \in \mathcal{N} \backslash n}\left(P_{n, n^{\prime}}-P_{n, n^{\prime}}^{\left(n^{\prime}\right)}\right)\right]^{+} .\right.
$$


The optimization problem (24) is still non-convex due to the convex function $e^{g_{n, n^{\prime}, j}}$ in the objective and concave function $\ln \left(\sum_{n^{\prime \prime} \in \mathcal{N}, n^{\prime \prime} \neq n} P_{n^{\prime \prime}, n^{\prime}}^{(n)}+P_{n, n^{\prime}}\right)$ in constraint $(d)$. We can use the first order approximation method [47], [48] as in (4) and (5) in order to convert the objective function and the constraint in (24) to convex forms.

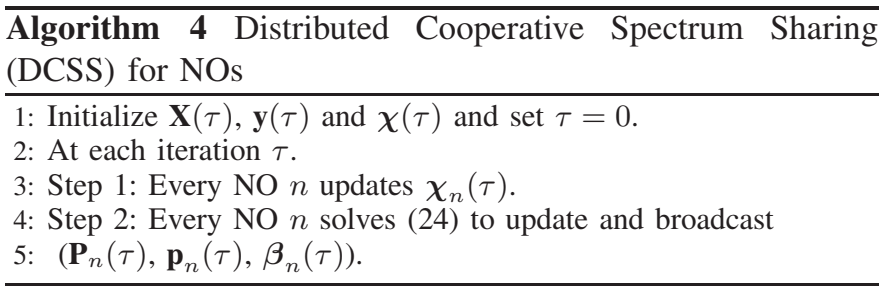

Algorithm 4 defines a distributed cooperative spectrum sharing between NOs when SSUs use a price-based interruption policy (PIP). Using DCSS (Algorithm 4), the NOs are able to optimize their profit through a cooperative game to reach the optimum point of their actions.

\section{SSUs as Followers}

Once a packet/call arrives at a SSU $j$ while it has already assigned all its available spectrum to SDUs, it chooses some SDUs in $\mathcal{L}_{j}$ to be interrupted depending on their corresponding NOs' price, incentive rate, and channel quality. The proposed utility function of every $\operatorname{SSU} j$ is given by

$$
\begin{aligned}
& \Pi_{j}\left(\mathbf{X}_{j}, \mathbf{y}_{j}\right) \\
& =U_{j}\left(\mathbf{X}_{j}, \mathbf{y}_{j}\right)+\sum_{n \in \mathcal{N}}\left\{-\sum_{n^{\prime} \in \mathcal{N}} P_{n^{\prime}, n} X_{j, n^{\prime}}+\sum_{n^{\prime} \in \mathcal{N}} P_{n, n^{\prime}} y_{j, n^{\prime}}\right. \\
& \left.\quad+\sum_{n^{\prime} \in \mathcal{N}} P_{n^{\prime}, n} \beta_{n^{\prime}, j}\left(1-\gamma_{j, n^{\prime}}^{\prime}\right) y_{j, n^{\prime}}\right\},
\end{aligned}
$$

where $U_{j}$ is a concave function reflecting the gain of SSU $j$ from using the spectrum for itself. Every SSU $j$ of NO $n$ should pay the cost $\sum_{n^{\prime} \in \mathcal{N}} P_{n^{\prime}, n} X_{j, n^{\prime}}$ to the NOs due to using a portion of the spectrum for itself. In the above, the term $\sum_{n^{\prime} \in \mathcal{N}} P_{n^{\prime}, n} \beta_{n^{\prime}, j}\left(1-\gamma_{j, n^{\prime}}^{\prime}\right) y_{j, n^{\prime}}$ is the amount of reimbursement for SSU $j$. The game among the SSUs can be formulated as the following optimization problem:

$$
\begin{aligned}
\text { Problem 5: } \max _{\mathbf{X}_{j}, \mathbf{y}_{j}} \Pi_{j}\left(\mathbf{X}_{j}, \mathbf{y}_{j}\right) \\
\text { s.t. } \quad a) \sum_{j \in \mathcal{J}} X_{j, n} \leq W_{n}, \forall n \in \mathcal{N}, \\
\text { b) } \sum_{n \in \mathcal{N}} y_{j, n} \leq \sum_{n \in \mathcal{N}} X_{j, n}, \forall j \in \mathcal{J} .
\end{aligned}
$$

Here, we have an optimization problem with a convex objective function and a coupled constraint $\sum_{j \in \mathcal{J}} X_{j, n} \leq W_{n}$. We form the Lagrangian of (27) in order to split the main problem into smaller local subproblems [50]. We take a dual decomposition by relaxing the coupling constraint in problem (27) to obtain:

$$
\begin{gathered}
\text { Problem 5-1: } \max _{\mathbf{X}_{j}, \mathbf{y}_{j}} \quad U_{j}\left(\mathbf{X}_{j}, \mathbf{y}_{j}\right)-\sum_{n \in \mathcal{N}} P_{n}\left(X_{j, n}-y_{j, n}\right) \\
+\sum_{n \in \mathcal{N}}\left\{P_{n} \beta_{n}\left(1-\gamma_{j} \frac{P_{n}}{\sum_{n^{\prime} \in \mathcal{N}} P_{n^{\prime}}}\right) y_{j, n^{\prime}}\right. \\
\left.\quad-\epsilon_{j, n}\left(X_{j, n}-W_{n}\right)\right\} \\
\text { s.t. } \quad \sum_{n \in \mathcal{N}} y_{j, n} \leq \sum_{n \in \mathcal{N}} X_{j, n}, \forall j \in \mathcal{J},
\end{gathered}
$$

where $\epsilon$ is a dual variable and the optimization problem can be locally solved by every SSU $j$. Every SSU $j$ uses Algorithm 5 to solve (28).

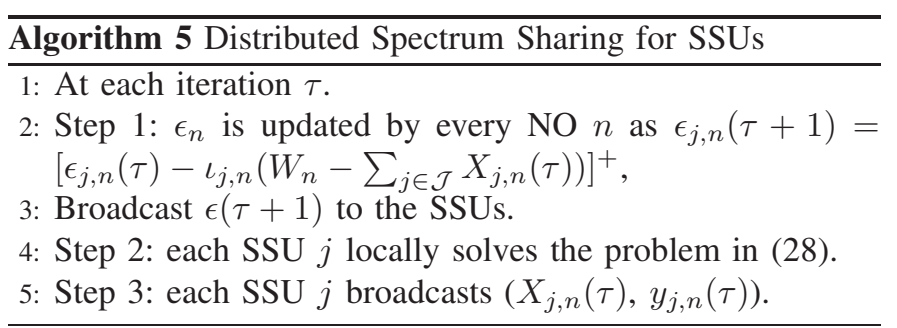

In Algorithm 5, every NO $n$ updates $\epsilon_{j, n}$ for every SSU $j$ at each iteration $t$. Then, it sends around $\epsilon(\tau+1)$. Every SSU $j$ uses the new value of $\epsilon_{j, n}(\tau+1)$ to solve the optimization problem (28). The optimal solutions of (28) are used in the next iterations. Note that step 1 in Algorithm 5 can be locally calculated at SSUs. So, the optimization problem (28) can be solved distributively by every SSU $j$ for a given set of prices and incentive rates.

\section{Multiple Operator Subgame Perfect Equilibrium (MOSPE)}

In the proposed multiple operator spectrum sharing model, the SPE is an equilibrium state where NOs and SSUs have no incentives to deviate from that point.

Theorem 3: Let $(\boldsymbol{P}, \boldsymbol{\beta})$ be a solution of (24) for NOs and $(\boldsymbol{D}, \boldsymbol{a})$ be a solution of (28) for SSUs. Then the point $\left(\boldsymbol{P}^{*}, \boldsymbol{\beta}^{*}, \boldsymbol{D}^{*}, \boldsymbol{a}^{*}\right)$ is a multiple operator SPE (MOSPE) point for the proposed model if and only if the following conditions hold for any possible solution $(\boldsymbol{P}, \boldsymbol{\beta}, \boldsymbol{D}, \boldsymbol{a})$.

$$
\text { a) } \begin{array}{ll}
\Pi\left(\boldsymbol{P}^{*}, \boldsymbol{\beta}^{*}, \boldsymbol{D}^{*}, \boldsymbol{a}^{*}\right) \geq \Pi\left(\boldsymbol{P}, \boldsymbol{\beta}, \boldsymbol{D}^{*}, \boldsymbol{a}^{*}\right), \\
\text { b) } \Pi_{j}\left(\boldsymbol{P}^{*}, \boldsymbol{\beta}^{*}, \boldsymbol{D}_{j}^{*}, \boldsymbol{a}_{j}^{*}, \boldsymbol{D}_{-j}^{*}, \boldsymbol{a}_{-j}^{*}\right) \geq \\
\Pi_{j}\left(\boldsymbol{P}^{*}, \boldsymbol{\beta}^{*}, \boldsymbol{D}_{j}, \boldsymbol{a}_{j}, \boldsymbol{D}_{-j}^{*}, \boldsymbol{a}_{-j}^{*}\right), \forall j \in \mathcal{J} .
\end{array}
$$

Proof: The first constraint implies that the NOs maximize their profit for a given $\mathbf{D}^{*}$ and $\mathbf{a}^{*}$ by finding optimum $\left(\mathbf{P}^{*}, \boldsymbol{\beta}^{*}\right)$. The second one defines the Nash equilibrium point for the noncooperative game between SSUs. In the equilibrium points, supplies and demands are equal and players will not leave those points.

We propose Algorithm 6 to solve the spectrum sharing in a multiple-operator scenario. 
$\overline{\text { Algorithm } 6 \text { Stackelberg Spectrum Sharing (S3) for Multiple }}$
Operators Scenario

1: Initialize $\mathbf{a}(\tau), \mathbf{D}(\tau)$ and set $\tau=0$.

2: At each iteration $\tau$.

3: Step 1: Every leader (NO) $n$ solves (24) to update and broadcast $\left(\mathbf{P}_{n}(\tau), \boldsymbol{\beta}_{n}(\tau)\right)$ Continue this procedure until reaches to the equilibrium point. Every leader (NO) $n$ broadcasts $\left(\mathbf{P}_{n}(\tau), \boldsymbol{\beta}(\tau)\right)$ of the equilibrium to SSUs.

4: Step 2: Every SSU (Follower) $j$ solves (28) to update and broadcast $\mathbf{a}_{j}(\tau)$ and $\mathbf{D}_{j}(\tau)$ Continue this procedure until attains to the equilibrium point Every SSU (Follower) $j$ broadcasts $\mathbf{a}_{j}(\tau)$ and $\mathbf{D}_{j}(\tau)$ of the equilibrium to leaders.

\section{LOW LATENCY AND NETWORK RELIABILITY}

User-centric distributed spectrum sharing (UDSS) is inherently opportunistic since SSUs share their unused spectrum with SDUs depending on their current traffic volume and offered incentive rate and price. Once SSUs reclaim their spectrum, they stop the ongoing SDUs' transmissions to start their own transmissions. This interruption probability depends on the operators' settings (e.g. price and incentive rate). Therefore, operators can control their coverage and outage probability by adjusting incentive and price. In other words, if an operator needs an Internet connectivity somewhere, a new SSU can be motivated to share its unused resources. Failing to convince users to share their resources results in spectrum outage. Based on this, we calculate the interruption probability (IP) and the spectrum outage probability (SOP) for single and muti-operator scenarios to investigate how NOs and users can benefit in such time-varying networks. These parameters are then used to analyze transmission latency and reliability of guaranteed latency. As before, we assume that the SSUs use TDMA scheme to share their spectrum with the SDUs. Furthermore, the SDUs and SSUs transmit a fixed-size data frame during each time-slot. Therefore, every spectrum sharing parameter, e.g. $X_{j}, Y_{j}$, and $d_{j, k}$ are in terms of the number of time-slots.

\section{A. Latency and Reliability in Single-Operator Scenario}

The IP should be investigated in more detail in order to improve the performance of spectrum sharing in DNA networks.

Definition 4: The average IP of an ongoing transmission of SDU $k$ in a given channel (time-slot) of $S S U j$ can be calculated by

$$
\gamma_{j, k}=\frac{d_{j, k}}{Y_{j}} \sum_{z=\left(X_{j}-Y_{j}\right)+1}^{\infty} \rho_{j} \frac{\kappa_{j}^{z} \exp \left(-\kappa_{j}\right)}{z !}
$$

where $\rho_{j}=\left\{\begin{array}{cc}\phi_{j} /\left|\mathcal{L}_{j}\right|, & \phi_{j}<\left|\mathcal{L}_{j}\right| \\ 1, & \phi_{j} \geq\left|\mathcal{L}_{j}\right|\end{array}\right.$ and $\phi_{j}=z-\left(X_{j}-\right.$ $\left.Y_{j}\right)$ represents the number of excessive packets arriving at $S S U j$. Parameter $\left|\mathcal{L}_{j}\right|$ denotes the size of $\mathcal{L}_{j}$ which is the set of SDUs using $S S U j$ and $\kappa_{j}^{z} \exp \left(-\kappa_{j}\right) / z$ ! is the probability of having $z$ packet arrivals following a Poisson distribution. Parameter $\kappa_{j}$ represents the packet arrival rate of SSU $j$. Instead of using Poisson distribution for new arrivals, in the calculation of interruption probabilities, the channel availability can be estimated by a learning process based on the real channel observations and predictions by Multi-Armed Bandits theory [51]. This is however out of the scope of this paper and should be dealt in a separate paper.

The probability that an allocated time-slot is used by SDU $k$ without an interruption by the SSU $j$, i.e. $z \leq\left(X_{j}-Y_{j}\right)$, is $1-$ $\gamma_{j, k}$. If SSU $j$ has more than $\left(X_{j}-Y_{j}\right)$ packet arrivals, it stops transmissions of SDU $k$ depending on $d_{j, k} / Y_{j}$. In the above equation, $\rho_{j}$ represents the average selection probability of the SDUs to be interrupted. The average interruption probability (AIP) can be calculated by

$$
\gamma_{j}=\sum_{k \in \mathcal{L}_{j}} \gamma_{j, k}
$$

In the second market, every SSU $j$ supplies the spectrum to the SDUs with probability $\left(1-\gamma_{j}\right)$. We define spectrum outage of SDUs for a DNA network as an event when none of SSUs inside a cluster can provide the spectrum during a period of time. We assume that each user can reach any of the SSUs in the cluster. In IoT networks one of the key performance merits is limited latency in the data transmission. For the given upper bound on the latency $(\delta)$ the network reliability is defined as a probability that the requirements for the limited latency will be met.

Definition 5: The spectrum outage probability (SOP) is the probability that none of the SSUs inside a cluster in DNA networks is able to provide at least one free channel during $\delta$ consecutive time-slots. We can calculate SOP for a DNA network as

$$
O_{\delta}=\prod_{j \in \mathcal{J}} \gamma_{j}^{\delta}
$$

So, the network reliability is defined as

$$
r_{\delta}=1-O_{\delta} .
$$

In a homogeneous scenario when the SSUs have equal arrival rates $\kappa$, the SOP can be calculated by $\gamma_{j}^{J \delta}$.

The average spectrum supplied by $\mathrm{SSU} j$ for SDUs can be calculated by

$$
Y_{j}=\sum_{z=0}^{X_{j}}\left(X_{j}-z\right) \frac{\kappa_{j}^{z} \exp \left(-\kappa_{j}\right)}{z !} .
$$

\section{B. Latency and Reliability in Multiple-Operator Scenario}

In a multiple-operator scenario, the SSUs have to decide about SDUs belonging to which NO should be interrupted once a new traffic arrives when all available spectrum has been assigned. Thus, every SSU can have different spectrum interruption preference level to the SDUs reflecting their corresponding NOs' prices and incentive rates.

Definition 6: The average interruption probability of ongoing transmissions of the SDUs belonging to $N O n^{\prime}$ in a given channel (time-slot) of SSU j of NO $n$ can be calculated by

$$
\gamma_{j, n}=\frac{\frac{P_{n^{\prime}, n}}{\beta_{n^{\prime}, j}}}{\sum_{n^{\prime \prime} \in \mathcal{N}} \frac{P_{n^{\prime \prime}, n}}{\beta_{n^{\prime \prime}, j}}} \sum_{z=\left(X_{j}-Y_{j}\right)+1}^{\infty} \rho_{j} \frac{\kappa_{j}^{z} \exp \left(-\kappa_{j}\right)}{z !} .
$$

The interruption probability $\gamma_{j, n}$ in a multiple-operator scenario not only depends on the traffic of SSU $j$ of NO $n$ but also incentive rate $\beta_{n^{\prime}, j}$ and price $P_{n^{\prime}, n}$ of NO $n^{\prime}$. In the above equation, $\mathrm{SSU} j$ drops with higher probability the ongoing transmissions of the SDUs belonging to the NO 
with a higher price and lower incentive rate. According to the proposed price and incentive-based interruption scheme (PIP), every SSU $j$ of NO $n$ supplies the spectrum

$$
Y_{j, n^{\prime}}=\frac{\frac{\beta_{n^{\prime}, j}}{P_{n^{\prime}, n}}}{\sum_{n^{\prime \prime} \in \mathcal{N}} \frac{\beta_{n^{\prime \prime}, j}}{P_{n^{\prime \prime}, n}}} \sum_{z=0}^{X_{j}}\left(X_{j}-z\right) \frac{\kappa_{j}^{z} \exp \left(-\kappa_{j}\right)}{z !}
$$

to SDUs of NO $n^{\prime}$.

In the SSU's side, the probability that an $\operatorname{SSU} j$ is selected by SDUs is given by

$$
S_{j}=\frac{\left(1-\gamma_{j}\right)}{\sum_{j^{\prime} \in \mathcal{J}}\left(1-\gamma_{j^{\prime}}\right)} \times \frac{a_{j}}{\sum_{j^{\prime} \in \mathcal{J}} a_{j^{\prime}}},
$$

where $0 \leq\left(1-\gamma_{j}\right) / \sum_{j^{\prime} \in \mathcal{J}}\left(1-\gamma_{j^{\prime}}\right) \leq 1$ and $0 \leq$ $a_{j} / \sum_{j^{\prime} \in \mathcal{J}} a_{j^{\prime}} \leq 1$ are the relative non-interruption probability and portion of the spectrum shared by SSU $j$, respectively. It means that SDUs prefer to select SSUs with lower IP and larger amount of shared spectrum.

In a multiple-operator scenario for a given latency threshold $\delta$, the average spectrum outage probability of every SDU belonging to NO $n$ is given by

$$
O_{n}=\prod_{n^{\prime} \in \mathcal{N} \backslash n} \prod_{j \in \mathcal{J}_{n^{\prime}}}\left(\gamma_{j, n^{\prime}}\right)^{\delta}
$$

and network reliability again as $r_{\delta}\{n\}=1-O_{n}$ with $\delta$ as the transmission latency threshold.

\section{ViI. Performance Evaluation}

In order to validate and analyze the performance of the proposed solutions, CVX tools are used to implement optimization problems in Matlab. We consider a network with three NOs and assume that their users are uniformly distributed within the simulated network, unless stated otherwise. To serve their users, the NOs deploy three different types of network infrastructure: FNI, HNI, and DNI (Fig. 5). Furthermore, we assume that the NO with FNI deploys at most five PAPs and the NO with HNI has two PAPs as its fixed part of infrastructure. For a single-operator scenario, only one of the possible strategies will be activated. Every NO owning $20 \mathrm{MHz}$ serves 5-20 SDUs in the network. Furthermore, we assume that the sharing rate $\alpha_{j}$ of SSU $j$ changes between 0.1 and 0.5 unless mentioned otherwise. According to the concept of DNA, this range of sharing rates needs to be investigated in more detail since very small sharing rate $\left(\alpha_{j}<0.1\right)$ is unable to make a big change in the network performance. Higher sharing rates $\left(\alpha_{j}>0.5\right)$ imply that most of the users share their free resources with SDUs in the network. The SSUs, PAPs and SDUs are randomly distributed throughout the network. In order to calculate the channel efficiency for SDUs, we only consider path-loss. First, we consider profit and utility of NO and SSUs under the proposed double Stackelberg game and optimization problems for both single and multi-operator scenarios. Finally, we investigate the network reliability to guarantee a given latency in the transmissions of the proposed spectrum sharing schemes for both single and multi-operator scenarios.

\section{A. Single Operator Scenario}

We analyze the proposed games and optimization problems in terms of profit of network operators and utility of both

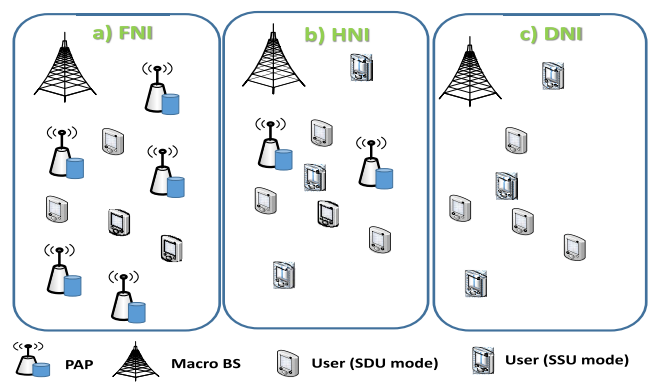

Fig. 5. Network infrastructure models,

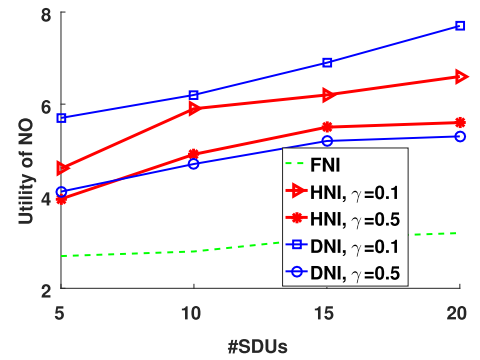

Fig. 6. Normalized Profit versus number of SDUs with $\gamma$ as a parameter.

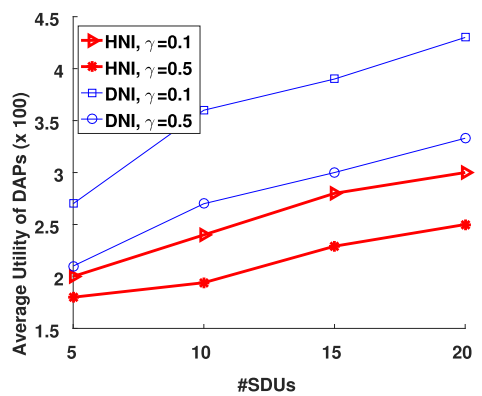

Fig. 7. Utility versus the number of SDUs with $\gamma$ as a parameter.

SSUs and SDUs. Figs. 6-7 are based on solving the proposed double Stackelberg game and optimization problems for single operator scenario, where every SSU with different traffic volumes competes with other SSUs to get enough spectrum for themselves and also to share it with the SDUs. Fig. 6 presents the normalized profit of the $\mathrm{NO}$ with different strategies versus the number of SDUs. We can see that deploying DNA concept by the NO significantly improves its profit by factor $200 \%$ and $250 \%$. According to this result, the DNI strategy is more suitable for any network with low-traffic users since they might have unused spectrum for sharing with other users. Fig. 7 demonstrates that the SSUs prefer to participate in spectrum sharing process of a NO with DNI strategy instead of HNI. Increasing the number of SSUs results in an improvement in the utility function. Depending on the traffic load of the SSUs, their average utility can be improved by choosing DNI between $50 \%$ and $60 \%$ in comparison to the HDI strategy.

The average utility per SDU versus the number of SDUs with $\gamma$ as a parameter is shown in Fig. 8. By increasing the number of SDUs, the available resources including the spectrum and the SSU should be shared among more SDUs so that the performance per user decreases. In the proposed model, SDUs try to choose the less utilized APs to have more reliable access. One can see from Fig. 8 that DNI strategy results in significant benefit for SDUs in comparison to FNI. 


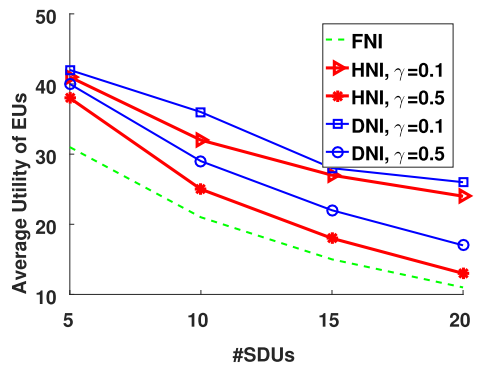

Fig. 8. Average utility per SDU versus the number of SDUs with $\gamma$ as a parameter (for $\iota=0.01$ ).

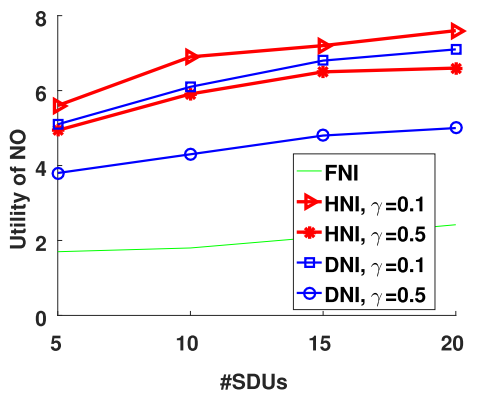

Fig. 9. Normalized Profit versus number of SDUs with $\gamma$ as a parameter

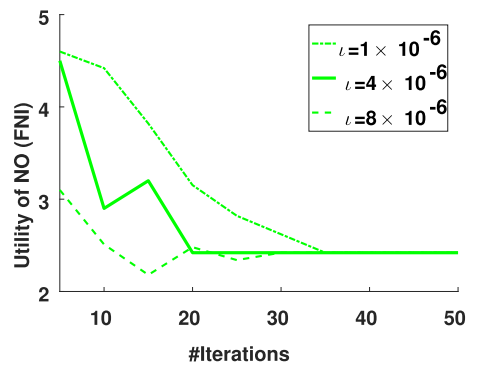

Fig. 10. Average utility versus the number of iterations with $\iota$ as a parameter

\section{B. Mutiple-Operator Scenario}

Fig. 9 is generated based on the proposed double Stackelberg game and the optimization problems for multi-operator scenario, where the SSUs with different traffic volumes and the NOs with different strategies compete with to improve their benefit. Fig. 9 presents profit of the NOs with different strategies versus the number of SDUs with $\gamma$ as parameter. We can see that deploying DNA concept in addition to a fixed infrastructure by the NOs significantly improves their profit. According to the result, the HNI strategy is more suitable for a NO in a competitive market. This dominant strategy can improve utility of the $\mathrm{NO}$ at least by factor three.

\section{Complexity and Convergence}

Figs. 10-12 show the number of iterations required to reach the equilibrium point by Algorithm 3. According to the figures, the proposed game among SSUs, SDUs and NO quickly (20 iterations) reaches the equilibrium point when the NO deploys FNI (see Fig. 10). The NO with strategies DNI and HNI requires at least 30 and 40 iterations, respectively, depending on the learning rate.

Fig. 13 shows the convergece result of a multi-operator scenario, where NOs deploy UDSS model to share their spectrum. According to the results, the proposed algorithm requires at least 80 iterations based on proper learning rate.

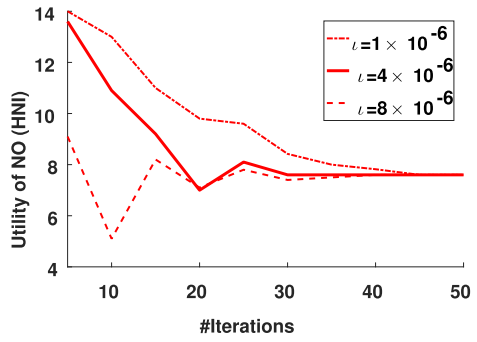

Fig. 11. Average utility versus the number of iterations with $\iota$ as a parameter.

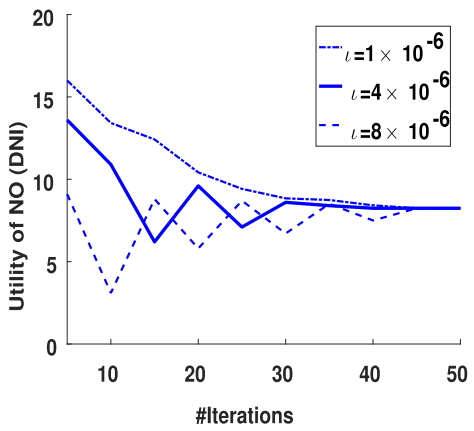

Fig. 12. Average utility versus the number of iterations with $\iota$ as a parameter

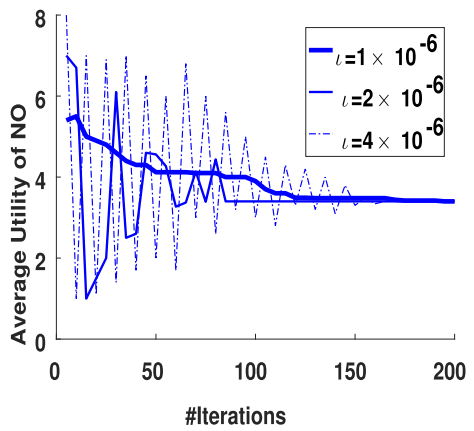

Fig. 13. Average utility versus the number of iterations with $\iota$ as a parameter.

The convergence of the proposed algorithms is achieved after a finite number of iterations and thus, Algorithms 1-5 are guaranteed to reach an equilibrium point. Due to their transmission range, the SDUs and SSUs can reach a limited number of users in their vicinity, and thus, the number of alternatives is finite. Furthermore, the proposed algorithms converge to the equilibrium points with a limited number of iterations. For the practical implementation of the algorithms, communication between players is required only for updating (per iteration) price, incentive rate, demands and sharing values, which can be done through the common control channel.

\section{Latency and Network Reliability}

In Fig. 14 for a NO with DNI strategy, one can see that the network reliability is improved by increasing the number of SSUs $J$ and non-availability interval measured in number of time-slots $\delta$. According to Fig. 14, an efficient DNA network even for delay-sensitive applications (real-time services with $\delta=1$ ) can simply be established if the NOs are able to introduce enough SSUs to the network. On the other hand, the DNA spectrum sharing model is very efficient for delaytolerant applications (services tolerating $\delta>1$ ). For the arrival rate $\kappa=0.9$ and $\delta=1$, the network reliability would be improved by $60 \%$ if the number of SSUs $J$ increases from 1 to 2 . 


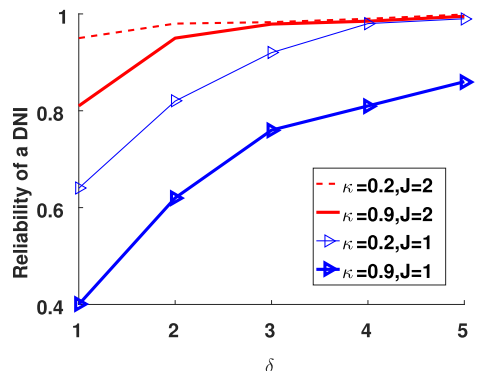

Fig. 14. The network reliability versus $\delta$ with arrival rate $\kappa$ and the number of SSUs $J$ as parameters.

Fig. 15 shows results for a simple scenario including two different network operators with equal incentive rate $\beta$. We assume that these NOs are sharing spectrum with together and the first operator (blue curves) increases its price between 1 and 5 while the second operator (red curves with triangles) has a fixed price equal to 2 . According to Fig. 15, an increase in the price results in a significant reduction in the network reliability of the first operator if only one SSU is used. On the other hand, introducing enough SSUs to the network reduces the spectrum outage probability significantly and increase network reliability. Notice that Fig. 15 shows the results for delay-sensitive applications $(\delta=1)$. With enough motivated SSUs in the network, NOs can improve network reliability for low latency services. In general, the spectrum outage (or reliability) depends on the network parameter setting. For instance in our setting, in Fig. 15, introducing one extra SSU can significantly reduce the spectrum outage probability in the network.

In order to show the selection probability of available SSUs, we assume that all SSUs except SSU $j$ have equal interruption probability $\bar{\gamma}=0.4$. Furthermore, every SSU shares spectrum $\bar{a}=2 \mathrm{MHz}$. Thus, we reformulate (36) for $\mathrm{SSU} j$ as

$$
S_{j}=\frac{1-\gamma_{j}}{\sum_{j^{\prime} \in \mathcal{J} \backslash j} 1-\bar{\gamma}_{j^{\prime}}+\left(1-\gamma_{j}\right)} \times \frac{a_{j}}{\sum_{j^{\prime} \in \mathcal{J} \backslash j} \bar{a}_{j^{\prime}}+a_{j}} .
$$

Fig. 16 shows the impact of the number of candidate SSUs $J$ and interruption probability $\gamma$ on the probability of selecting a given SSU $j$ by an SDU. As expected, the SSUs with lower interruption probability have the higher selection probability. Furthermore, the SSUs can significantly increase their selection probability by increasing their sharing rate $a_{j}$. Obviously, the number of competitors (SSUs) can affect the selection probability. Therefore, the SSUs with lower interruption probability have more chances to increase their selection probability by adjusting their sharing rates even when there are many competing SSUs. For the number of SSUs $J=5$ the selection probability of given SSU $j$ increases by a factor of 10 if the interruption probability $\gamma_{j}$ reduces from 0.7 to 0.2 .

In summary, our analysis shows that the NOs and SSUs can benefit from deploying the user-centric spectrum sharing model depending on different adjustments of network parameters, e.g. $\alpha, \beta$ and prices. Higher demands by SSUs result in a significant improvement of NOs' profits while there is an optimum demand for every SSU. The number of SSUs significantly enhances the profit while it moderately improves the SDU's utility. The convergence of proposed algorithms for user-centric spectrum sharing model depend on the adjustment of the learning rate.

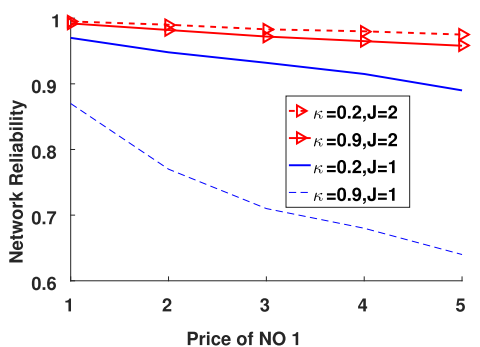

Fig. 15. The network reliability for a two-operator scenario versus price with $\beta_{n}=0.5, \kappa=0.9, P_{2}=2$, and $\delta=1$.

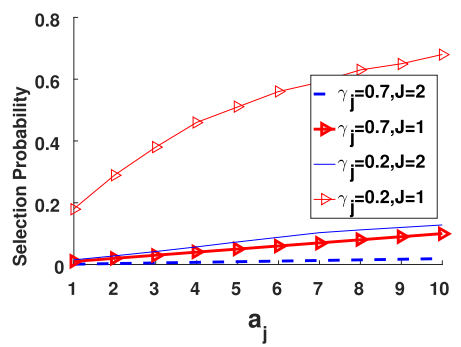

Fig. 16. The selection probability of a candidate $\operatorname{SSU} j$ (38) versus the shared spectrum $a_{j}$ with $\bar{\gamma}=0.4$ and $\bar{a}=2$.

\section{CONCLUSION}

We have developed and analyzed a new user-centric networking paradigm for ubiquitous spectrum sharing mechanism where every user can share and use the spectrum. The proposed user-centric spectrum sharing mechanism enables users to share their unused resources independent of time and location. A game theoretic solution is used to model and solve the proposed problem for single and multipleoperator scenarios. In addition, we have developed a dynamic strategy to deal with the uncertainty of traffic in the spectrum sharing process by the SDUs where SSUs may occasionally reclaim their shared spectrum. Also, a price and incentivebased interruption policy for SSUs has been proposed, where every SSU interrupts the SDUs' transmissions according to the price and incentive rates of their corresponding NOs. We analyze network latency and reliability of UDSS under uncertainty of traffic patterns of SSUs to show that DNA always can be an option for the network operators to extend their network coverage and capacity.

The numerical results show that the UDSS model, depending on different settings, can improve at least 100\% and 30\% profit and utility for NOs and SDUs, respectively. Furthermore, network reliability is significantly improved depending on the network parameters for SSUs and NOs. The numerical results indicate a significant improvement in utilities of NOs, and SSUs if they apply our model in DNA.

As future work, we will consider co-channel interference in the spectrum sharing mechanism in order to increase frequency reuse in DNA networks.

\section{REFERENCES}

[1] L. A. Lopes, R. Sofia, H. Haci, and H. Zhu, "A proposal for dynamic frequency sharing in wireless networks," IEEE/ACM Trans. Netw., vol. 24, no. 5, pp. 2621-2633, Oct. 2016.

[2] Y. Wang and X. Lin, "User-provided networking for QoE provisioning in mobile networks," IEEE Wireless Commun., vol. 22, no. 4, pp. 26-33, Apr. 2015. 
[3] A. S. Shafigh et al., "A framework for dynamic network architecture and topology optimization," IEEE/ACM Trans. Netw., vol. 24, no. 2, pp. 717-730, Apr. 2016.

[4] G. Iosifidis, L. Gao, J. Huang, and L. Tassiulas, "Incentive mechanisms for user-provided networks," IEEE Commun. Mag., vol. 52, no. 9, pp. 20-27, Sep. 2014.

[5] V. G. Rolla and M. Curado, "Enabling wireless cooperation in user provided networks," in Proc. IFIP/IEEE Int. Symp. Integr. Netw. Manage. (IM), May 2015, pp. 1114-1121.

[6] A. S. Shafigh and S. Glisic, "User-centric spectrum sharing in dynamic network architecture," in Proc. IEEE Globecom Workshops (GC Wkshps), Dec. 2016, pp. 1-7.

[7] L. Gao, G. Iosifidis, J. Huang, and L. Tassiulas, "Hybrid data pricing for network-assisted user-provided connectivity," in Proc. IEEE INFOCOM, Apr./May 2014, pp. 682-690.

[8] M. M. Khalili, L. Gao, J. Huang, and B. H. Khalaj, "Incentive design and market evolution of mobile user-provided networks," in Proc. IEEE Conf. Comput. Commun. Workshops (INFOCOM WKSHPS), Apr./May 2015, pp. 498-503.

[9] (May 21, 2016). Fon Wireless Ltd. [Online]. Available: https://corp.fon.com/en

[10] (May 21, 2016). Karma. [Online]. Available: https://yourkarma.com

[11] (May 21, 2016). Open Garden. [Online]. Available: https:// opengarden.com

[12] (May 21, 2016). HotSpot. [Online]. Available: https://play.google.com/store/apps/details?id=org.eeiiaa.airmobs

[13] G. Iosifidis, L. Gao, J. Huang, and L. Tassiulas, "Enabling crowd-sourced mobile Internet access," in Proc. IEEE INFOCOM, Apr./May 2014, pp. 451-459.

[14] Q. Ma, L. Gao, Y.-F. Liu, and J. Huang, "A game-theoretic analysis of user behaviors in crowdsourced wireless community networks," in Proc. 13th Int. Symp. Modeling. Optim. Mobile, Ad Hoc, Wireless Netw. (WiOpt), May 2015, pp. 355-362.

[15] M. H. Afrasiabi and R. Guérin, "Exploring user-provided connectivity," IEEE/ACM Trans. Netw., vol. 24, no. 1, pp. 542-554, Feb. 2016.

[16] M. Yildiz, M. A. Khan, F. Sivrikaya, and S. Albayrak, "Cooperation incentives based load balancing in UCN: A probabilistic approach," in Proc. IEEE Global Commun. Conf. (GLOBECOM), Dec. 2012, pp. 2746-2752.

[17] H. Eghbali and V. W. S. Wong, "Bandwidth allocation and pricing for SDN-enabled home networks," in Proc. IEEE Int. Conf. Commun. (ICC), Jun. 2015, pp. 5342-5347.

[18] S. E. Taş̧̧i and Ö. Korçak, "Incentivizing user provided connectivity for enhanced quality of service," in Proc. IEEE 27th Annu. Int. Symp. Pers., Indoor, Mobile Radio Commun. (PIMRC), Sep. 2016, pp. 1-7.

[19] M. H. Afrasiabi and R. Guérin, "Pricing strategies for userprovided connectivity services," in Proc. IEEE Conf. Comput. Commun. (INFOCOM), Mar. 2012, pp. 2766-2770.

[20] G. Iosifidis, L. Gao, J. Huang, and L. Tassiulas. (2017). "Efficient and fair collaborative mobile Internet access." [Online]. Available: https://arxiv.org/abs/1612.05129

[21] A. Kostopoulos and M. Papadopouli, "Looking for new allies in mobile Internet market," in Proc. IEEE Conf. Comput. Commun. Workshops (INFOCOM), Apr. 2016, pp. 239-244.

[22] Y. Zhang, Y. Gu, L. Liu, M. Pan, Z. Dawy, and Z. Han, "Incentive mechanism in crowdsourcing with moral hazard," in Proc. IEEE Wireless Commun. Netw. Conf. (WCNC), Mar. 2015, pp. 2085-2090.

[23] M. Tang, L. Gao, H. Pang, J. Huang, and L. Sun, "A multi-dimensional auction mechanism for mobile crowdsourced video streaming," in Proc. 14th Int. Symp. Modeling. Optim. Mobile, Ad Hoc, Wireless Netw. (WiOpt), May 2016, pp. 1-8.

[24] L. Gao, M. Tang, H. Pang, J. Huang, and L. Sun, "Performance bound analysis for crowdsourced mobile video streaming," in Proc. Annи. Conf. Inf. Sci. Syst. (CISS), Mar. 2016, pp. 366-371.

[25] N. Do, Y. Zhao, C.-H. Hsu, and N. Venkatasubramanian, "Crowdsourced mobile data transfer with delay bound," ACM Trans. Internet Technol., vol. 16, no. 4, 2016, Art. no. 28.

[26] B. Lorenzo and F. J. Gonzalez-Castano, "A matching game for data trading in operator-supervised user-provided networks," in Proc. IEEE Int. Conf. Commun. (ICC), May 2016, pp. 1-7.

[27] B. Lorenzo, F. Gomez-Cuba, J. Garcia-Rois, F. J. Gonzalez-Castano, and J. C. Burguillo, "A microeconomic approach to data trading in user provided networks," in Proc. IEEE Globecom Workshops (GC Wkshps), Dec. 2015, pp. 1-7.
[28] H. Haci, H. Zhu, and J. Wang, "Resource allocation in user-centric wireless networks," in User-Centric Networking. Cham, Switzerland: Springer, 2014, pp. 197-208.

[29] B. A. A. Nunes, M. A. S. Santos, B. T. de Oliveira, C. B. Margi, K. Obraczka, and T. Turletti, "Software-defined-networking-enabled capacity sharing in user-centric networks," IEEE Commun. Mag., vol. 52, no. 9, pp. 28-36, Sep. 2014.

[30] Q. Ma, L. Gao, Y.-F. Liu, and J. Huang, "Economic analysis of crowdsourced wireless community networks," IEEE Trans. Mobile Comput., vol. 16, no. 7, pp. 1856-1869, Jul. 2016.

[31] P. Ostovari, J. Wu, and A. Khreishah, "Cooperative mobile Internet access with opportunistic scheduling," in Proc. Resilience Week (RWS), Aug. 2015, pp. 1-6.

[32] P. Ostovari, J. Wu, and A. Khreishah, "Cooperative Internet access using helper nodes and opportunistic scheduling," IEEE Trans. Veh. Technol., vol. 66, no. 7, pp. 6439-6448, Jul. 2017.

[33] D. Giatsios, G. Iosifidis, and L. Tassiulas, "Mobile edge-networking architectures and control policies for 5G communication systems," in Proc. 14th Int. Symp. Modeling Optim. Mobile, Ad Hoc, Wireless Netw. (WiOpt), May 2016, pp. 1-8.

[34] M. Ishino, Y. Koizumi, and T. Hasegawa, "Leveraging proximity services for relay device discovery in user-provided IoT networks," in Proc. IEEE 2nd World Forum Internet Things (WF-IoT), Dec. 2015, pp. 553-558.

[35] B. Lorenzo, F. J. Gonzalez-Castano, and Y. Fang, "A novel collaborative cognitive dynamic network architecture," IEEE Trans. Wireless Commun., vol. 24, no. 1, pp. 74-81, Feb. 2017.

[36] D. Syrivelis, G. Iosifidis, D. Delimpasis, K. Chounos, T. Korakis, and L. Tassiulas, "Bits and coins: Supporting collaborative consumption of mobile Internet," in Proc. IEEE Conf. Comput. Commun. (INFOCOM), Apr./May 2015, pp. 2146-2154.

[37] I. Baumgart and F. Hartmann, "Towards secure user-centric networking: Service-oriented and decentralized social networks," in Proc. 5th IEEE Conf. Self-Adapt. Self-Organizing Syst. Workshops (SASOW), Oct. 2011, pp. 3-8.

[38] A. Zúquete and C. Frade, "Fast VPN mobility across Wi-Fi hotspots," in Proc. 2nd Int. Workshop Secur. Commun. Netw. (IWSCN), May 2010, pp. $1-7$.

[39] M. Yildiz and M. A. Khan, "Mobility behavior modeling in UCN," in Proc. IEEE Wireless Commun. Netw. Conf. (WCNC), Apr. 2013, pp. 2184-2189.

[40] D. Niyato and E. Hossain, "A microeconomic model for hierarchical bandwidth sharing in dynamic spectrum access networks," IEEE Trans. Comput., vol. 59, no. 7, pp. 865-877, Jul. 2010.

[41] D. Yang et al., "Joint optimization for coordinated configuration of product families and supply chains by a leader-follower Stackelberg game," Eur. J. Oper. Res., vol. 246, no. 1, pp. 263-280, 2015.

[42] K. Zhu and E. Hossain, "Joint mode selection and spectrum partitioning for device-to-device communication: A dynamic Stackelberg game," IEEE Trans. Wireless Commun., vol. 14, no. 3, pp. 1406-1420, Mar. 2015.

[43] S. Haddadi and A. Ghasemi, "Pricing-based Stackelberg game for spectrum trading in self-organised heterogeneous networks," IET Commun., vol. 10, no. 11, pp. 1374-1383, Jul. 2016.

[44] M. Yu and S. H. Hong, "A real-time demand-response algorithm for smart grids: A Stackelberg game approach," IEEE Trans. Smart Grid, vol. 7, no. 2, pp. 879-888, Mar. 2016.

[45] R. J. Aumann, "Backward induction and common knowledge of rationality," Games Econ. Behav., vol. 8, no. 1, pp. 6-19, 1995.

[46] I. Arieli and R. J. Aumann, "The logic of backward induction," J. Econ. Theory, vol. 159, pp. 443-464, Sep. 2015.

[47] A. Tolli, H. Pennanen, and P. Komulainen, "Decentralized minimum power multi-cell beamforming with limited backhaul signaling," IEEE Trans. Wireless Commun., vol. 10, no. 2, pp. 570-580, Feb. 2011.

[48] J. Kaleva, A. Tölli, and M. Juntti, "Decentralized sum rate maximization with QoS constraints for interfering broadcast channel via successive convex approximation," IEEE Trans. Signal Process., vol. 64, no. 11, pp. 2788-2802, Jun. 2016.

[49] H. Terelius, U. Topcu, and R. M. Murray, "Decentralized multi-agent optimization via dual decomposition," IFAC Proc. Volumes, vol. 44, no. 1, pp. 11245-11251, Jan. 2011

[50] C. W. Tan, D. P. Palomar, and M. Chiang, "Distributed optimization of coupled systems with applications to network utility maximization," in Proc. IEEE Int. Conf. Acoust. Speech Signal Process., vol. 5, May 2006, p. 5.

[51] S. Maghsudi and E. Hossain, "Multi-armed bandits with application to 5G small cells," IEEE Wireless Commun., vol. 23, no. 3, pp. 64-73, Jun. 2016 